\title{
Synthesis of Some $N$-[(4-Substituted-1-Piperazinyl)-Oxo(Alkyl and Ethylcarbamoyl)]-3-(2-Thiophenyl)Acrylamides as Non-Steroidal Anti-Allergic and Anti-Inflammatory Agents
}

\author{
Osama I. Abd El-Salam ${ }^{*}$, Ahmed M. Shalaby ${ }^{2}$, Abdulah A. El-Sawy ${ }^{3}$, \\ Soha Elshihaby ${ }^{2}$, Mohamed Abdulla ${ }^{4}$ \\ ${ }^{1}$ Department of Applied Organic Chemistry, National Research Centre, Cairo, Egypt \\ ${ }^{2}$ Department of Peptide Chemistry, National Research Centre, Cairo, Egypt \\ ${ }^{3}$ Department of Chemistry, Faculty of Science, Benha University, Banha, Egypt \\ ${ }^{4}$ Research Units, Hi-Care Pharmaceutical Co., Cairo, Egypt \\ Email: *oiel_salam@yahoo.com
}

Received November 18, 2012; revised January 9, 2013; accepted January 26, 2013

Copyright (C) 2013 Osama I. Abd El-Salam et al. This is an open access article distributed under the Creative Commons Attribution License, which permits unrestricted use, distribution, and reproduction in any medium, provided the original work is properly cited.

\begin{abstract}
An interesting approach for the design of anti-allergies is rationally considered. It was proved that current anti-allergic drugs comprise piperazine and acrylic acid segments. In harmony with these findings, new products 5a-u were synthesized starting from conjugated 2-thiopheneacrylic acid with amino acid esters 3a-g followed by coupling of their acid derivatives 4a-g with some piperazine segments, with the aim to increase their biological activities and decrease side effects. The anti-allergic and anti-inflammatory activities of the products were evaluated and promising results were obtained.
\end{abstract}

Keywords: Thiophene; Amino Acids; Piperazines; Anti-Allergy; Anti-Inflammatory

\section{Introduction}

The clinical manifestations of the allergic reaction are elicited by the subsequent interaction of the mediators with the end organ smooth muscle or mucous membranes. Traditionally symptomatic treatment of allergies and bronchial asthma has been provided by $\beta$-sympathomimetic agents, methylxanthines, corticosteroids, and anticholinergics [1-3]. Japanese authors have synthesized a series of acrylamide derivatives linked with different piperazines via alkyl chains and proved them as promising anti-allergic candidates (Figure 1(A)) [4-7].

It is known that amino acids and peptides are being taxonomically natural, physiologically nontoxic and chemically multifunctional, their rationalized conjugates with biologically active synthetic organic agents, are hypothesized to be more potent, particularly less toxic than their parent biologicals [8]. Further, thiophene derivatives have received interest because of their potential pharmacological utility and their incorporation in synthetic peptides [9-11]. Several thiophene derivatives are

*Corresponding author. known and have been used as antibiotic, anti-allergic, anti-migraine, antioxidant agents [12-14].

In the present work, we have attempted to blend some of the foregoing considerations with our own experience and synthetic techniques, to design compounds [B] with an architectural resemblance to (Figure 1(B)) [15-17]. Thus, some amino acid residues are acting as a bridge between 2-thiopheneacrylic acid and some piperazine derivatives to get the two hypothetical anti-allergic segments i.e. thiophene acrylamide and piperazine. The presence of the amino acid residues introduce an additional hydrogen bond acceptor site (i.e., $\mathrm{C}=\mathrm{O}$ ) that could represent a new structural contribution for the anti-allergic profile of these compounds.

\section{Experimental}

\subsection{Instruments}

Melting points were determined in open glass capillaries using an Electrothermal IA 9000 SERIES digital melting point apparatus (Electrothermal, UK) and are uncorrected. Optical rotations were measured by a "Polax-D" po- 
<smiles>[R1]C=CC(=O)NCCN1CCN([R2])CC1</smiles>

[A]<smiles>[R]N1CCN(C(=O)NC(=O)/C=C/c2cccs2)CC1</smiles>

[B]

$$
\begin{aligned}
\mathrm{n} & =1-4 \\
\mathrm{R}_{1} & =\text { aryl or heteraryl } \\
\mathrm{R}_{2} & =\text { alkyl or aryl }
\end{aligned}
$$

$$
\begin{aligned}
& \mathrm{R}=\text { amino acid residue } \\
& \mathrm{R}_{2}=\text { alkyl, ester or aryl }
\end{aligned}
$$

Figure 1. Structure design of the target products [B].

larimeter (ATAGO), provided by a SI-Na-1 Sodium lamp. Microanalyses were performed with all final compounds on an Elementar-Vario EL (Elementar-Vario EL, Germany) (Micro-analytical Unit, Central Services Laboratory, National Research Centre, Cairo; Egypt). The ${ }^{1} \mathrm{H}-\mathrm{NMR}$ spectra were recorded on a Varian Mercury VX-300 NMR spectrometer (Varian, USA). ${ }^{1} \mathrm{H}-\mathrm{NMR}$ spectra were run at $300 \mathrm{MHz}$ in $\mathrm{DMSO}-\mathrm{d}_{6}$ as solvent. Chemical shifts $\delta$ are quoted in ppm and were related to that of the solvents. Mass spectra were recorded on a Shimadzu GCMS-QP 1000EX (EI, 70 eV) (Shimadzu, Japan) and Hewlett-Packard (EI, 70 eV) (Hewlett- Packard, USA). IR spectra were obtained with a BruckerVector 22 (Bruker Rhein-Stetten, Germany). All the reactions were monitored using thin layer chromatography (TLC) using silica gel aluminum sheets $60 \mathrm{~F}_{254}$ (Merck). While "S" stands for a chromatographic solvent system of chloroform/methanol/acetic acid, $85 / 10 / 5$ by volume, $\mathrm{S}_{1}$ and $\mathrm{S}_{2}$ represent the same solvent system to which petroleum ether $\left(40^{\circ} \mathrm{C}-60^{\circ} \mathrm{C}\right)$ was added, in an equal or half ratio volume respectively.

\subsection{Organic Preparations}

\subsubsection{Preparation of $E$-3-(thiophen-2-yl)acrylamido) esters (3a-g) \\ General Procedures}

1) Acid Chloride Method [Procedure A]

Triethylamine $(2.42 \mathrm{~g} \equiv 3.4 \mathrm{ml}, 24 \mathrm{mmol})$ was added in portions to a well stirred cold solution $\left(0^{\circ} \mathrm{C}-5^{\circ} \mathrm{C}\right)$ of thiophene-2-carbonyl chloride 2 [freshly prepared from thiophene-2-acrylic acid $(1.85 \mathrm{~g}, 12 \mathrm{mmol})]$ and amino acid ester hydrochloride (12 mmol) in THF/DCM (1:4, $150 \mathrm{ml})$. The reaction mixture was stirred for $3 \mathrm{~h}$ at the same temperature with simultaneous adjustment of $\mathrm{pH} \approx$ 8. The TEA hydrochloride was filtered off, excess solvent distilled off and residue in, mainly, ethyl acetate (EtOAc) was washed with $\mathrm{HCl}(1 \mathrm{~N}, 3 \times 20 \mathrm{ml})$, Na$\mathrm{HCO}_{3}(5 \%, 3 \times 20 \mathrm{ml}), \mathrm{H}_{2} \mathrm{O}(3 \times 20 \mathrm{ml})$ and dried over anhydrous sodium sulfate, excess solvent was distilled off to get $3 \mathrm{a}, \mathrm{b}$ and $3 \mathrm{~g}$ in good yields (Table 1).
2) In Situ Active Ester Method [Procedure B]

A solution of DCCI $(3 \mathrm{~g}, 15 \mathrm{mmol})$ in THF $(10 \mathrm{ml})$ was added, over $30 \mathrm{~min}$., to a stirred cold solution $\left(0^{\circ} \mathrm{C}\right.$ $\left.5^{\circ} \mathrm{C}\right)$ of thiophene-2-acrylic acid $(2.31 \mathrm{~g}, 15 \mathrm{mmol})$ and $\mathrm{N}$-hydroxybenzotiazole (HOBt) $(1.7 \mathrm{~g}, 15 \mathrm{mmol})$ in $\mathrm{THF}$ $(50 \mathrm{ml})$. Stirring was continued for $15 \mathrm{~min}$. at the same temperature, and a mixture of the corresponding amino acid or dipeptide ester hydrochloride $(15 \mathrm{mmol})$ and TEA $(1.5 \mathrm{~g} \equiv 2.13 \mathrm{ml}, 15 \mathrm{mmol})$ in THF $(50 \mathrm{ml})$ was then added. Stirring was continued at the same temperature for $3 \mathrm{~h}$, at $\mathrm{pH} \approx 8\left(\mathrm{Et}_{3} \mathrm{~N}\right)$ and at $0^{\circ} \mathrm{C}$ overnight, then at room temperature for $24 \mathrm{~h}$. Drops of glacial acetic acid were added to the cold $\left(0^{\circ} \mathrm{C}\right)$ suspension and the reaction mixture was filtered off. The filtrate was evaporated, and the residue was taken in acetonitrile $(10 \mathrm{ml})$, then, kept in cold, and the formed urea (DCHU) was separated by filtration. Solvent was distilled off under reduced pressure and the residue was taken up in EtOAc, washed with $\mathrm{NaHCO}_{3}(1 \mathrm{~N}, 3 \times 10 \mathrm{ml})$, distilled water $(3 \times 10 \mathrm{ml})$, $\mathrm{KHSO}_{4}(5 \%, 3 \times 10 \mathrm{ml})$, distilled water $(3 \times 10 \mathrm{ml})$ and dried over anhydrous sodium sulfate. The dried and decolorized EtOAc solution was concentrated $(5 \mathrm{ml})$ and chromatographically purified on manually prepared preparative TLC plates to get the products (3a-g).

The same procedure was undertaken to couple the acid products $4 \mathrm{a}-\mathrm{g}$ with the appropriate substituted piperazines to afford $5 \mathrm{a}-\mathrm{u}$.

3) Mixed Anhydride Method [Procedure C]

Ethyl chloroformate $(1.08 \mathrm{~g} \equiv 0.95 \mathrm{ml}, 10 \mathrm{mmol})$ was added to a stirred and cold $\left(-15^{\circ} \mathrm{C}\right)$ THF solution of 3-(2thienyl)acrylic acid (1.54 gm, $10 \mathrm{mmol})$ and $N$-methylmorpholine $(\sim 1 \mathrm{ml}, 10 \mathrm{mmol})$. The reaction mixture was stirred for additional 10 minutes, and then cold solution of free valine methyl ester or glycylglycine ethyl ester (10 mmol) in THF $(30 \mathrm{ml})$ was added. Stirring was maintained for $3 \mathrm{~h}$ at $\left(-15^{\circ} \mathrm{C}\right)$ then for $12 \mathrm{~h}$ at room temperature. The solvent was evaporated and the residue was taken into EtOAc $(30 \mathrm{ml})$ to be washed with $\mathrm{KHSO}_{4}(3 \%$, $3 \times 10)$, distilled water $(3 \times 10 \mathrm{ml}), \mathrm{NaHCO}_{3}(3 \%, 3 \times$ $10)$, and finally with water $(3 \times 10 \mathrm{ml})$ then dried over anhydrous sodium sulfate. The solvent was evaporated 
Table 1. Physical data of $E$-3-(thiophen-2-yl)acrylamido)esters (3a-g).

\begin{tabular}{|c|c|c|c|c|c|c|c|c|}
\hline \multirow[b]{2}{*}{ Compd. No. } & \multirow{2}{*}{ Yield, (\%) [Procedure] } & \multirow{2}{*}{ M.p. $\left({ }^{\circ} \mathrm{C}\right)$} & \multirow{2}{*}{ Mol formula (M. Wt.) } & \multicolumn{4}{|c|}{ Analysis (Calcd/Found) } & \multirow{2}{*}{$\mathrm{R}_{f} \times 100$ (Eluent) } \\
\hline & & & & $\mathrm{C}$ & $\mathrm{H}$ & $\mathrm{N}$ & $\mathrm{S}$ & \\
\hline $3 a$ & $\begin{array}{l}79[\mathrm{~A}] \\
26[\mathrm{~B}]\end{array}$ & $172-174$ & $\mathrm{C}_{11} \mathrm{H}_{13} \mathrm{NO}_{3} \mathrm{~S} 239.29$ & $\begin{array}{l}55.21 \\
55.11\end{array}$ & $\begin{array}{l}5.48 \\
5.42\end{array}$ & $\begin{array}{l}5.85 \\
5.92\end{array}$ & $\begin{array}{l}13.40 \\
13.32\end{array}$ & $\begin{array}{l}65 \\
S_{2}\end{array}$ \\
\hline $3 b$ & $\begin{array}{l}91[\mathrm{~A}] \\
64[\mathrm{C}]\end{array}$ & $145-147$ & $\mathrm{C}_{11} \mathrm{H}_{13} \mathrm{NO}_{3} \mathrm{~S} 239.27$ & $\begin{array}{l}55.21 \\
55.09\end{array}$ & $\begin{array}{l}5.48 \\
5.38\end{array}$ & $\begin{array}{l}5.85 \\
5.98\end{array}$ & $\begin{array}{l}13.40 \\
13.46\end{array}$ & $\begin{array}{l}59 \\
\mathrm{~S}_{2}\end{array}$ \\
\hline $3 \mathrm{c}$ & $68[\mathrm{~B}]$ & $77-80$ & $\mathrm{C}_{12} \mathrm{H}_{15} \mathrm{NO}_{3} \mathrm{~S} 253.32$ & $\begin{array}{l}56.90 \\
56.76\end{array}$ & $\begin{array}{l}5.97 \\
5.85\end{array}$ & $\begin{array}{l}5.53 \\
5.67\end{array}$ & $\begin{array}{l}12.66 \\
12.74\end{array}$ & $\begin{array}{l}60 \\
S_{1}\end{array}$ \\
\hline $3 d$ & $59[\mathrm{~B}]$ & $94-96$ & $\mathrm{C}_{13} \mathrm{H}_{17} \mathrm{NO}_{3} \mathrm{~S} 267.34$ & $\begin{array}{l}58.41 \\
58.29\end{array}$ & $\begin{array}{l}6.41 \\
6.33\end{array}$ & $\begin{array}{l}5.24 \\
5.14\end{array}$ & $\begin{array}{l}11.99 \\
11.91\end{array}$ & $\begin{array}{l}65 \\
S_{1}\end{array}$ \\
\hline $3 e$ & $47[\mathrm{~B}]$ & $103-105$ & $\mathrm{C}_{17} \mathrm{H}_{17} \mathrm{NO}_{3} \mathrm{~S} 315.39$ & $\begin{array}{l}64.74 \\
64.58\end{array}$ & $\begin{array}{l}5.43 \\
5.31\end{array}$ & $\begin{array}{l}4.44 \\
4.56\end{array}$ & $\begin{array}{l}10.17 \\
10.26\end{array}$ & $\begin{array}{l}47 \\
S_{2}\end{array}$ \\
\hline $3 \mathrm{f}$ & $63[\mathrm{~B}]$ & $130-132$ & $\mathrm{C}_{17} \mathrm{H}_{17} \mathrm{NO}_{4} \mathrm{~S} 331.39$ & $\begin{array}{l}61.61 \\
61.47\end{array}$ & $\begin{array}{l}5.17 \\
5.09\end{array}$ & $\begin{array}{l}4.23 \\
4.35\end{array}$ & $\begin{array}{l}9.68 \\
9.56\end{array}$ & $\begin{array}{l}52 \\
\mathrm{~S}_{1}\end{array}$ \\
\hline $3 g$ & $\begin{array}{l}59[\mathrm{~A}] \\
39[\mathrm{C}]\end{array}$ & $119-121$ & $\mathrm{C}_{13} \mathrm{H}_{16} \mathrm{~N}_{2} \mathrm{O}_{4} \mathrm{~S} 296.34$ & $\begin{array}{l}52.69 \\
52.57\end{array}$ & $\begin{array}{l}5.44 \\
5.35\end{array}$ & $\begin{array}{l}9.45 \\
9.61\end{array}$ & $\begin{array}{l}10.82 \\
10.70\end{array}$ & $\begin{array}{c}66 \\
S\end{array}$ \\
\hline
\end{tabular}

to dryness and the obtained residue triturated with nhexane. The obtained solid was collected by filtration and crystallized from ethanol/n-hexane to give the products $3 \mathrm{~d}$ and $3 \mathrm{~g}$ as identified by melting point and TLC in comparison with samples prepared according to procedure B.

The same procedure was undertaken to couple the acid products $4 \mathrm{a}, 4 \mathrm{~d}, 4 \mathrm{f}$ and $4 \mathrm{~g}$ with the appropriate substituted piperazines to afford $5 \mathrm{a}, 5 \mathrm{c}, 5 \mathrm{k}, 5 \mathrm{p}, 5 \mathrm{~s}$ and $5 \mathrm{u}$.

\subsubsection{Preparation of $E$-3-(thiophen-2-yl)acrylamido) acids (4a-g) \\ General Procedure}

The KOH solution ( $30 \mathrm{ml}, 0.1 \mathrm{~N}$ ) was drop-wisely added to a stirred solution cold $\left(-5^{\circ} \mathrm{C}-0^{\circ} \mathrm{C}\right)$ of $3 \mathrm{a}-\mathrm{g}(10 \mathrm{mmol})$ in methanol $(20 \mathrm{ml})$. Then, the reaction mixture was stirred at same temperature for $1 \mathrm{~h}$, at room temperature for $24 \mathrm{~h}$ and evaporated under vacuum till dryness. The obtained solid was dissolved in distilled water $(50 \mathrm{ml})$, cooled in an ice bath, stirred, acidified with $\mathrm{HCl}(0.1 \mathrm{~N})$ to $\mathrm{pH} \sim 2-3$ and the precipitate was separated by filtration, washed with distilled water, dried to afford the acids 4a-g (Table 2).

\subsubsection{Synthesis of $N$-[(4-substituted-1-piperazinyl)- oxo-(alkyl and ethylcarbamoyl)]-3-pheny- acrylamides (5a-u)}

1) (E)-N-(2-(4-methylpiperazin-1-yl)-2-oxoethyl)-3-(thiophen-2-yl)acrylamide (5a)

TLC: $R_{f}=0.35$ [S $\mathrm{S}_{1}$, yield: $42 \%$ (Procedure B), 53\% (Procedure C), m.p. $170^{\circ} \mathrm{C}-172^{\circ} \mathrm{C}$. IR $\vee\left(\mathrm{cm}^{-1}\right): 3338$ (NH), 1657 ( $\mathrm{C}=\mathrm{O}$ amide). ${ }^{1} \mathrm{H}$ NMR (DMSO- $\left.d_{6}, \delta, \mathrm{ppm}\right)$ : $2.13\left(\mathrm{~s}, 3 \mathrm{H}, \mathrm{NCH}_{3}\right), 2.27$ - 2.39 (m, 4H, 2CH , Pip-H), $2.41-3.51$ (m, 4H, 2CH 2 , Pip-H), 4.01 (s, 2H, $\mathrm{COCH}_{2}$ ), $6.51(\mathrm{~d}, \mathrm{~J}=15 \mathrm{~Hz}, 1 \mathrm{H},=\mathrm{CHCO}), 6.98(\mathrm{t}, \mathrm{J}=3.5 \mathrm{~Hz}, 1 \mathrm{H}$, Th-H4), 7.15 (d, J = 5 Hz, 1H, Th-H3), 7.50 (d, J = 15
$\mathrm{Hz}, 1 \mathrm{H}, \mathrm{CH}=\mathrm{CH}), 7.56(\mathrm{~d}, \mathrm{~J}=5 \mathrm{~Hz}, 1 \mathrm{H}, \mathrm{Th}-\mathrm{H} 5), 8.18$ (s, 1H, NH, exchangeable with $\left.\mathrm{D}_{2} \mathrm{O}\right)$. Mass: m/z 294 $\left([\mathrm{M}+1]^{+}, 12 \%\right)$. Calcd. for $\mathrm{C}_{14} \mathrm{H}_{19} \mathrm{~N}_{3} \mathrm{O}_{2} \mathrm{~S}$ (293.38). Calcd. C 57.31, H 6.53, N 14.32, S 10.93. Found: C 57.22, H $6.45, \mathrm{~N} 14.22, \mathrm{~S} 10.86$.

2) $(E)-N$-(2-(4-ethoxycarbonylpiperazin-1-yl)-2-oxoethyl)-3-(thiophen-2-Yl)acrylamide (5b)

TLC: $\left.\mathrm{R}_{\mathrm{f}}=0.55\left[\mathrm{~S}_{2}\right)\right]$, yield: $47 \%$ (Procedure B), m.p. $93^{\circ} \mathrm{C}-95^{\circ} \mathrm{C}$. IR $v\left(\mathrm{~cm}^{-1}\right): 3450(\mathrm{NH}), 1694(\mathrm{C}=\mathrm{O}$ ester $)$, $1663\left(\mathrm{C}=\mathrm{O}\right.$ amide). ${ }^{1} \mathrm{H}$ NMR (DMSO-d $\left.\mathrm{d}_{6}, \delta, \mathrm{ppm}\right): 1.26(\mathrm{t}$, $\left.\mathrm{J}=7.5 \mathrm{~Hz}, 3 \mathrm{H}, \mathrm{CH}_{2} \mathrm{CH}_{3}\right), 3.6-3.63\left(\mathrm{~m}, 4 \mathrm{H}, 2 \mathrm{CH}_{2}\right.$, Pip-H), 3.67-3.7 (m, 4H, 2 $\mathrm{CH}_{2}$, Pip-H), 4.1 (s, 2H, $\mathrm{COCH}_{2}$ ), 4.3 (q, J = 7.5 Hz, 2H, $\left.\mathrm{CH}_{2} \mathrm{CH}_{3}\right), 6.44$ (d, J = $16 \mathrm{~Hz}, 1 \mathrm{H},=\mathrm{CHCO}), 6.98$ (t, J = $3.5 \mathrm{~Hz}, 1 \mathrm{H}, \mathrm{Th}-\mathrm{H} 4)$, 7.29 (d, J = $5 \mathrm{~Hz}, 1 \mathrm{H}$, Th-H3), 7.47 (d, J = $16 \mathrm{~Hz}, 1 \mathrm{H}$, $\mathrm{CH}=\mathrm{CH}), 7.71(\mathrm{~d}, \mathrm{~J}=5 \mathrm{~Hz}, 1 \mathrm{H}, \mathrm{Th}-\mathrm{H} 5), 8.43$ (s, 1H, NH, exchangeable with $\left.\mathrm{D}_{2} \mathrm{O}\right)$. Mass: $\mathrm{m} / \mathrm{z} 352\left([\mathrm{M}+1]^{+}, 7 \%\right)$. Calcd. for $\mathrm{C}_{16} \mathrm{H}_{21} \mathrm{~N}_{3} \mathrm{O}_{4} \mathrm{~S}$ (351.42). Calcd. C 54.68, H 6.02, $\mathrm{N} 11.96, \mathrm{~S}$ 9.12. Found: C, 54.56, H 5.96, N 12.96, S 9.00 .

3) (E)-N-(2-(4-(4-fluorophenyl)piperazin-1-yl)-2-oxoethyl)3-(thiophen-2-yl)acryl-amide $(5 \mathrm{c})$

TLC: $\mathrm{R}_{\mathrm{f}}=0.45\left[\mathrm{~S}_{1}\right.$ ], yield: $53 \%$ (Procedure B), 65\% (Procedure C); m.p. $120^{\circ} \mathrm{C}-122^{\circ} \mathrm{C}$. IR $v\left(\mathrm{~cm}^{-1}\right): 3370$ $(\mathrm{NH}), 1670\left(\mathrm{C}=\mathrm{O}\right.$ amide). ${ }^{1} \mathrm{H}$ NMR (DMSO-d $\left.\mathrm{d}_{6}, \delta, \mathrm{ppm}\right)$ : 2.37 - 2.47 (m, 4H, 2CH $\mathrm{CH}_{2}$ Pip-H), 3.35 - 3.46 (m, 4H, $\left.2 \mathrm{CH}_{2}, \mathrm{Pip}-\mathrm{H}\right), 3.96$ (s, 2H, $\left.\mathrm{COCH}_{2}\right), 6.23$ - 6.33 (m, 2H, $\mathrm{Ph}-\mathrm{H}), 6.84$ (d, J = $16 \mathrm{~Hz}, 1 \mathrm{H},=\mathrm{CHCO}), 6.99$ - 7.05 (m, 2H, Ph-H), 7.06 (t, J = 3.5 Hz, 1H, Th-H4), 7.19 (d, J = 5 $\mathrm{Hz}, 1 \mathrm{H}, \mathrm{Th}-\mathrm{H} 3), 7.3$ (d, J = $16 \mathrm{~Hz}, 1 \mathrm{H}, \mathrm{CH}=\mathrm{CH}), 7.4$ (d, $\mathrm{J}=5 \mathrm{~Hz}, 1 \mathrm{H}$, Th-H5), $8.14(\mathrm{~s}, 1 \mathrm{H}, \mathrm{NH}$, exchangeable with $\left.\mathrm{D}_{2} \mathrm{O}\right)$. Mass: $\mathrm{m} / \mathrm{z} 374\left([\mathrm{M}+1]^{+}, 14 \%\right)$. Calcd. For $\mathrm{C}_{19} \mathrm{H}_{20} \mathrm{FN}_{3} \mathrm{O}_{2} \mathrm{~S}$ (373.44). Calcd. C 61.11, H 5.40, N 11.25, S 8.59. Found: C 60.98, H 5.32, N 11.39, S 8.67.

4) (E)- $N$-(3-(4-methylpiperazin-1-yl)-3-oxopropyl)-3-(thiophen-2-yl)acrylamide (5d) 
Table 2. Physical data of $E$-3-(thiophen-2-yl)acrylamido)acids (4a-g).

\begin{tabular}{|c|c|c|c|c|c|c|c|c|}
\hline \multirow[b]{2}{*}{ Compd. No. } & \multirow{2}{*}{ Yield (\%) } & \multirow{2}{*}{ M.p. $\left({ }^{\circ} \mathrm{C}\right)$} & \multirow{2}{*}{$\begin{array}{l}\text { Mol formula } \\
\text { (M. Wt.) }\end{array}$} & \multicolumn{4}{|c|}{ Analysis (Calcd/Found) } & \multirow{2}{*}{$\mathrm{R}_{f} \times 100$ (Eluent) } \\
\hline & & & & $\mathrm{C}$ & $\mathrm{H}$ & $\mathrm{N}$ & S & \\
\hline $4 a$ & 66 & $218-220$ & $\begin{array}{c}\mathrm{C}_{9} \mathrm{H}_{9} \mathrm{NO}_{3} \mathrm{~S} \\
211.24\end{array}$ & $\begin{array}{l}51.17 \\
51.09\end{array}$ & $\begin{array}{l}4.29 \\
4.21\end{array}$ & $\begin{array}{l}6.63 \\
6.72\end{array}$ & $\begin{array}{l}15.18 \\
15.14\end{array}$ & $\begin{array}{l}43 \\
S_{2}\end{array}$ \\
\hline $4 \mathrm{~b}$ & 72 & $167-169$ & $\begin{array}{c}\mathrm{C}_{16} \mathrm{H}_{21} \mathrm{~N}_{3} \mathrm{O}_{4} \mathrm{~S} \\
351.42\end{array}$ & $\begin{array}{l}54.68 \\
54.62\end{array}$ & $\begin{array}{l}6.02 \\
5.96\end{array}$ & $\begin{array}{l}11.96 \\
11.98\end{array}$ & $\begin{array}{l}9.12 \\
9.07\end{array}$ & $\begin{array}{l}47 \\
S_{2}\end{array}$ \\
\hline $4 \mathrm{c}$ & 65 & $125-127$ & $\begin{array}{c}\mathrm{C}_{11} \mathrm{H}_{13} \mathrm{NO}_{3} \mathrm{~S} \\
239.29\end{array}$ & $\begin{array}{l}55.21 \\
55.09\end{array}$ & $\begin{array}{l}5.48 \\
5.40\end{array}$ & $\begin{array}{l}5.85 \\
5.99\end{array}$ & $\begin{array}{l}13.40 \\
13.44\end{array}$ & $\begin{array}{l}45 \\
S_{1}\end{array}$ \\
\hline $4 d$ & 67 & $120-123$ & $\begin{array}{c}\mathrm{C}_{12} \mathrm{H}_{15} \mathrm{NO}_{3} \mathrm{~S} \\
253.36\end{array}$ & $\begin{array}{l}56.90 \\
56.74\end{array}$ & $\begin{array}{l}5.97 \\
5.89\end{array}$ & $\begin{array}{l}5.53 \\
5.72\end{array}$ & $\begin{array}{l}12.66 \\
12.58\end{array}$ & $\begin{array}{l}47 \\
S_{1}\end{array}$ \\
\hline $4 \mathrm{e}$ & 78 & $134-136$ & $\begin{array}{c}\mathrm{C}_{16} \mathrm{H}_{15} \mathrm{NO}_{3} \mathrm{~S} \\
301.36\end{array}$ & $\begin{array}{l}63.77 \\
63.51\end{array}$ & $\begin{array}{l}5.02 \\
4.94\end{array}$ & $\begin{array}{l}4.65 \\
4.79\end{array}$ & $\begin{array}{l}10.64 \\
10.56\end{array}$ & $\begin{array}{l}32 \\
\mathrm{~S}_{2}\end{array}$ \\
\hline $4 \mathrm{f}$ & 60 & $169-171$ & $\begin{array}{c}\mathrm{C}_{16} \mathrm{H}_{15} \mathrm{NO}_{4} \mathrm{~S} \\
317.37\end{array}$ & $\begin{array}{l}60.55 \\
60.41\end{array}$ & $\begin{array}{l}4.76 \\
4.68\end{array}$ & $\begin{array}{l}4.41 \\
4.53\end{array}$ & $\begin{array}{l}10.10 \\
9.96\end{array}$ & $\begin{array}{l}34 \\
S_{1}\end{array}$ \\
\hline $4 \mathrm{~g}$ & 84 & $190-192$ & $\begin{array}{c}\mathrm{C}_{11} \mathrm{H}_{12} \mathrm{~N}_{2} \mathrm{O}_{4} \mathrm{~S} \\
268.29\end{array}$ & $\begin{array}{l}49.24 \\
49.10\end{array}$ & $\begin{array}{l}4.51 \\
4.43\end{array}$ & $\begin{array}{l}10.44 \\
10.58\end{array}$ & $\begin{array}{l}11.95 \\
11.83\end{array}$ & $\begin{array}{c}38 \\
\mathrm{~S}\end{array}$ \\
\hline
\end{tabular}

TLC: $\mathrm{R}_{\mathrm{f}}=0.37$ [S $\mathrm{S}_{2}$ ], yield: $39 \%$ (Procedure B), m.p. $102^{\circ} \mathrm{C}-104^{\circ} \mathrm{C}$. IR $v\left(\mathrm{~cm}^{-1}\right): 3376(\mathrm{NH}), 1669(\mathrm{C}=\mathrm{O}$ amide). ${ }^{1} \mathrm{H}$ NMR (DMSO-d $\left.\mathrm{d}_{6}, \delta, \mathrm{ppm}\right): 2.23$ (s, $3 \mathrm{H}, \mathrm{NCH}_{3}$ ), $2.27\left(\mathrm{t}, \mathrm{J}=7.2 \mathrm{~Hz}, 2 \mathrm{H}, \mathrm{COCH}_{2}\right), 2.54-2.57(\mathrm{~m}, 4 \mathrm{H}$, $2 \mathrm{CH}_{2}$, Pip-H), 3.1 (t, J = 7.2 Hz, 2H, $\left.\mathrm{NHCH}_{2}\right), 3.28-3.31$ (m, 4H, 2CH 2 , Pip-H), 6.67 (d, J = 15.6 Hz, $1 \mathrm{H},=\mathrm{CHCO}$ ), $7.05(\mathrm{t}, \mathrm{J}=3.6 \mathrm{~Hz}, 1 \mathrm{H}, \mathrm{Th}-\mathrm{H} 4), 7.35(\mathrm{~d}, \mathrm{~J}=5.1 \mathrm{~Hz}, 1 \mathrm{H}$, Th-H3), $7.52(\mathrm{~d}, \mathrm{~J}=15.8 \mathrm{~Hz}, 1 \mathrm{H}, \mathrm{CH}=\mathrm{CH}), 7.63(\mathrm{~d}, \mathrm{~J}=$ $5.2 \mathrm{~Hz}, 1 \mathrm{H}, \mathrm{Th}-\mathrm{H} 5), 8.38(\mathrm{~s}, 1 \mathrm{H}, \mathrm{NH}$, exchangeable with $\left.\mathrm{D}_{2} \mathrm{O}\right)$. Mass: $\mathrm{m} / \mathrm{z} 308\left([\mathrm{M}+1]^{+}, 7 \%\right)$. Calcd. for $\mathrm{C}_{15} \mathrm{H}_{21} \mathrm{~N}_{3} \mathrm{O}_{2} \mathrm{~S}$ (307.41). Calcd. C 58.61, H 6.89, N 13.67, S 10.43. Found: C 58.47, H 6.81, N 13.79, S 10.51.

5) (E)- $N$-(3-(4-ethoxycarbonylpiperazin-1-yl)-3-oxopropyl)-3-(thiophen-2-Yl)acryl-amide (5e)

TLC: $\mathrm{R}_{\mathrm{f}}=0.48\left[\mathrm{~S}_{2}\right]$, yield: $47 \%$ (Procedure B), m.p. $113^{\circ} \mathrm{C}-115^{\circ} \mathrm{C}$. IR $v\left(\mathrm{~cm}^{-1}\right): 3319(\mathrm{NH}), 1714(\mathrm{C}=\mathrm{O}$ ester), 1657 (C=O amide). ${ }^{1} \mathrm{H}$ NMR (DMSO-d $\left.\mathrm{d}_{6}, \delta, \mathrm{ppm}\right)$ : $1.17\left(\mathrm{t}, \mathrm{J}=7.7 \mathrm{~Hz}, 3 \mathrm{H}, \mathrm{CH}_{2} \mathrm{CH}_{3}\right), 2.53(\mathrm{t}, \mathrm{J}=7.1 \mathrm{~Hz}, 2 \mathrm{H}$, $\left.\mathrm{COCH}_{2}\right), 3.16\left(\mathrm{t}, \mathrm{J}=7 \mathrm{~Hz}, 2 \mathrm{H}, \mathrm{NHCH}_{2}\right), 3.48-3.51(\mathrm{~m}$, $4 \mathrm{H}, 2 \mathrm{CH}_{2}$, Pip-H), $3.71-3.73\left(\mathrm{~m}, 4 \mathrm{H}, 2 \mathrm{CH}_{2}, \mathrm{Pip}-\mathrm{H}\right)$, 4.06 (q, J = 7.6 Hz, 2H, $\left.\mathrm{CH}_{2} \mathrm{CH}_{3}\right), 6.36(\mathrm{~d}, \mathrm{~J}=16.9 \mathrm{~Hz}$, $1 \mathrm{H},=\mathrm{CHCO}), 7.1(\mathrm{t}, \mathrm{J}=3.5 \mathrm{~Hz}, 1 \mathrm{H}, \mathrm{Th}-\mathrm{H} 4), 7.36(\mathrm{~d}, \mathrm{~J}=$ $5.1 \mathrm{~Hz}, 1 \mathrm{H}, \mathrm{Th}-\mathrm{H} 3), 7.62(\mathrm{~d}, \mathrm{~J}=17 \mathrm{~Hz}, 1 \mathrm{H}, \mathrm{CH}=\mathrm{CH})$, $7.68(\mathrm{~d}, \mathrm{~J}=5.1 \mathrm{~Hz}, 1 \mathrm{H}, \mathrm{Th}-\mathrm{H} 5), 8.46(\mathrm{~s}, 1 \mathrm{H}, \mathrm{NH}$, exchangeable with $\left.\mathrm{D}_{2} \mathrm{O}\right)$. Mass: $\mathrm{m} / \mathrm{z} 366\left([\mathrm{M}+1]^{+}, 11 \%\right)$. Calcd. for $\mathrm{C}_{17} \mathrm{H}_{23} \mathrm{~N}_{3} \mathrm{O}_{4} \mathrm{~S}$ (365.45). Calc. C 55.87, H 6.34, N 11.50, S 8.77. Found: C 55.73, H 6.27, N 11.64, S 8.86 .

6) (E)- $N$-(3-(4-(4-fluorophenyl)piperazin-1-yl)-3-oxopropyl)-3-(thiophen-2-yl)acryl-amide (5f)

TLC: $\mathrm{R}_{\mathrm{f}}=0.42\left[\mathrm{~S}_{1}\right]$, yield: $45 \%$ (Procedure B), m.p. $139^{\circ} \mathrm{C}-141^{\circ} \mathrm{C}$. IR $v\left(\mathrm{~cm}^{-1}\right): 3317(\mathrm{NH}), 1662(\mathrm{C}=\mathrm{O}$ amide). ${ }^{1} \mathrm{H}$ NMR (DMSO-d $\left.6, \delta, \mathrm{ppm}\right): 2.38-2.4(\mathrm{~m}, 2 \mathrm{H}$, $\left.\mathrm{COCH}_{2}\right), 2.98-3.0\left(\mathrm{~m}, 2 \mathrm{H}, \mathrm{NHCH}_{2}\right), 3.11-3.14(\mathrm{~m}, 4 \mathrm{H}$, $2 \mathrm{CH}_{2}$, Pip-H), 3.19 - 3.22 (m, 4H, $2 \mathrm{CH}_{2}$, Pip-H), 6.26 $6.35(\mathrm{~m}, 2 \mathrm{H}, \mathrm{Ph}-\mathrm{H}), 6.78(\mathrm{~d}, \mathrm{~J}=16 \mathrm{~Hz}, 1 \mathrm{H},=\mathrm{CHCO})$, 6.98 - 7.03 (m, 2H, Ph-H), 7.08 (d, J = $3 \mathrm{~Hz}, 1 \mathrm{H}, \mathrm{Th}-\mathrm{H} 4)$, $7.12(\mathrm{~d}, \mathrm{~J}=5 \mathrm{~Hz}, 1 \mathrm{H}, \mathrm{Th}-\mathrm{H} 3), 7.35$ (d, J = 5 Hz, 1H, Th-H5), 7.55 (d, J = $16 \mathrm{~Hz}, 1 \mathrm{H}, \mathrm{CH}=\mathrm{CH}), 8.12(\mathrm{~s}, 1 \mathrm{H}$, $\mathrm{NH}$, exchangeable with $\left.\mathrm{D}_{2} \mathrm{O}\right)$. Mass: $\mathrm{m} / \mathrm{z} 387\left([\mathrm{M}]^{+}\right.$, $100 \%$ ). Calcd. for $\mathrm{C}_{20} \mathrm{H}_{22} \mathrm{FN}_{3} \mathrm{O}_{2} \mathrm{~S}$ (387.47). Calcd. C 62.00, H 5.72, N 10.84, S 8.28. Found: C 62.12, H 5.82, $\mathrm{N} 10.72$, S 8.36.

7) (E)- $N$-(4-(4-methylpiperazin-1-yl)-4-oxobutyl)-3-(thiophen-2-Yl)acrylamide (5g)

TLC: $\mathrm{R}_{\mathrm{f}}=0.48\left[\mathrm{~S}_{1}\right]$, yield: $53 \%$ (Procedure B), m.p. $182^{\circ} \mathrm{C}-184^{\circ} \mathrm{C}$. IR $v\left(\mathrm{~cm}^{-1}\right): 3368(\mathrm{NH}), 1669(\mathrm{C}=\mathrm{O}$ amide). ${ }^{1} \mathrm{H}$ NMR (DMSO-d $\mathrm{d}_{6}, \delta$, ppm): $1.75-1.77$ (m, 2H, $\mathrm{CH}_{2} \mathrm{CH}_{2} \mathrm{CH}_{2}$ ), 2.21 (s, 3H, NCH$), 2.23\left(\mathrm{~m}, 2 \mathrm{H}, \mathrm{CH}_{2} \mathrm{CO}\right.$ ), 2.52 - 2.54 (m, 4H, 2CH 2 , Pip-H), 3.21 (m, $2 \mathrm{H}, \mathrm{NHCH}_{2}$ ), 3.25 - 3.27 (m, 4H, 2CH $\mathrm{CH}_{2}$ Pip-H), 6.42 (d, J = 16 Hz, 1H, $=\mathrm{CHCO}), 6.99(\mathrm{t}, \mathrm{J}=3.2 \mathrm{~Hz}, 1 \mathrm{H}, \mathrm{Th}-\mathrm{H} 4), 7.17(\mathrm{~d}, \mathrm{~J}=$ $5.1 \mathrm{~Hz}, 1 \mathrm{H}, \mathrm{Th}-\mathrm{H} 3), 7.55(\mathrm{~d}, \mathrm{~J}=5.1 \mathrm{~Hz}, 1 \mathrm{H}, \mathrm{Th}-\mathrm{H} 5)$, $7.62(\mathrm{~d}, 1 \mathrm{H}, \mathrm{J}=16 \mathrm{~Hz}, 1 \mathrm{H}, \mathrm{CH}=\mathrm{CH}), 8.14(\mathrm{~s}, 1 \mathrm{H}, \mathrm{NH}$, exchangeable with $\left.\mathrm{D}_{2} \mathrm{O}\right)$. Mass: $\mathrm{m} / \mathrm{z} 322\left([\mathrm{M}+1]^{+}, 12 \%\right)$. Calcd. for $\mathrm{C}_{16} \mathrm{H}_{23} \mathrm{~N}_{3} \mathrm{O}_{2} \mathrm{~S}$ (321.44). Calcd. C, 59.78; $\mathrm{H}$, 7.21; N, 13.07; S, 9.98. Found: C, 59.75; H, 9.17; N, $13.12 ; \mathrm{S}, 9.94$.

8) (E)- $N$-(4-(4-ethoxycarbonylpiperazin-1-yl)-4-oxobutyl)-3-(thiophen-2-Yl)acrylamide (5h)

TLC: $\mathrm{R}_{\mathrm{f}}=0.43$ [S $\mathrm{S}_{2}$ ], yield: $39 \%$ (Procedure B), m.p. $121^{\circ} \mathrm{C}-123^{\circ} \mathrm{C}$. IR $v\left(\mathrm{~cm}^{-1}\right): 3338(\mathrm{NH}), 1695(\mathrm{C}=\mathrm{O}$ ester), $1660\left(\mathrm{C}=\mathrm{O}\right.$ amide). ${ }^{1} \mathrm{H}$ NMR (DMSO- $\left.\mathrm{d}_{6}, \delta, \mathrm{ppm}\right)$ : $1.17\left(\mathrm{t}, \mathrm{J}=7.2 \mathrm{~Hz}, 3 \mathrm{H}, \mathrm{CH}_{2} \mathrm{CH}_{3}\right), 1.79-1.82(\mathrm{~m}, 2 \mathrm{H}$, $\left.\mathrm{CH}_{2} \mathrm{CH}_{2} \mathrm{CH}_{2}\right), 2.53\left(\mathrm{~m}, 2 \mathrm{H}, \mathrm{CH}_{2} \mathrm{CO}\right), 3.16(\mathrm{~m}, 2 \mathrm{H}$, $\mathrm{NHCH}_{2}$ ), 3.46 - 3.48 (m, 4H, $2 \mathrm{CH}_{2}$, Pip-H), 3.79 - 3.82 (m, 4H, 2CH 2 Pip-H), 4.25 (q, 2H, $\left.\mathrm{CH}_{2} \mathrm{CH}_{3}\right), 6.46$ (d, J $=16 \mathrm{~Hz}, 1 \mathrm{H},=\mathrm{CHCO}), 7.07(\mathrm{t}, \mathrm{J}=3.2 \mathrm{~Hz}, 1 \mathrm{H}, \mathrm{Th}-\mathrm{H} 4)$, $7.4(\mathrm{~d}, \mathrm{~J}=5 \mathrm{~Hz}, 1 \mathrm{H}, \mathrm{Th}-\mathrm{H} 3), 7.54(\mathrm{~d}, 1 \mathrm{H}, \mathrm{J}=16 \mathrm{~Hz}, 1 \mathrm{H}$, $\mathrm{CH}=\mathrm{CH}), 7.62(\mathrm{~d}, \mathrm{~J}=5 \mathrm{~Hz}, 1 \mathrm{H}, \mathrm{Th}-\mathrm{H} 5), 8.23(\mathrm{~s}, 1 \mathrm{H}, \mathrm{NH}$, exchangeable with $\left.\mathrm{D}_{2} \mathrm{O}\right)$. Mass: $\mathrm{m} / \mathrm{z} 380\left([\mathrm{M}+1]^{+}, 2 \%\right)$. Calcd. for $\mathrm{C}_{18} \mathrm{H}_{25} \mathrm{~N}_{3} \mathrm{O}_{4} \mathrm{~S}$ (379.47). Calcd. C 56.97, H 6.64, N 11.07, S 8.45. Found: C 57.09; H 6.76, N 10.95, S 8.53 . 
9) (E)-N-(4-(4-(4-fluorophenyl)piperazin-1-yl)-4-oxobutyl)-3-(thiophen-2-yl)acryl-amide (5i)

TLC: $\mathrm{R}_{\mathrm{f}}=0.39\left[\mathrm{~S}_{1}\right]$, yield: $55 \%$ (Procedure B), m.p. $157^{\circ} \mathrm{C}-159^{\circ} \mathrm{C}$. IR $v\left(\mathrm{~cm}^{-1}\right): 3317(\mathrm{NH}), 1662(\mathrm{C}=\mathrm{O}$ amide). ${ }^{1} \mathrm{H}$ NMR (DMSO-d $\left.\mathrm{d}_{6}, \delta, \mathrm{ppm}\right): 1.85-1.87(\mathrm{~m}, 2 \mathrm{H}$, $\mathrm{CH}_{2} \mathrm{CH}_{2} \mathrm{CH}_{2}$ ), 2.24 - 2.27 (m, $2 \mathrm{H}, \mathrm{CH}_{2} \mathrm{CO}$ ), 2.98-3.0 (m, $\left.2 \mathrm{H}, \mathrm{NHCH}_{2}\right), 3.22-3.24\left(\mathrm{~m}, 4 \mathrm{H}, 2 \mathrm{CH}_{2}, \mathrm{Pip}-\mathrm{H}\right)$, 3.39-3.42 (m, 4H, 2CH $\mathrm{CH}_{2}$ Pip-H), 6.43 (d, J = $17 \mathrm{~Hz}, 1 \mathrm{H}$, $=\mathrm{CHCO}), 6.65(\mathrm{~m}, 2 \mathrm{H}, \mathrm{Ph}-\mathrm{H}), 6.98(\mathrm{~m}, 2 \mathrm{H}, \mathrm{Ph}-\mathrm{H}), 7.12$ $(\mathrm{d}, \mathrm{J}=3.1 \mathrm{~Hz}, 1 \mathrm{H}, \mathrm{Th}-\mathrm{H} 4), 7.17(\mathrm{~J}=5 \mathrm{~Hz}, 1 \mathrm{H}, \mathrm{Th}-\mathrm{H} 3)$, 7.37 (d, J = 5.1 Hz, 1H, Th-H5), 7.57 (d, 1H, J = $17 \mathrm{~Hz}$, $1 \mathrm{H}, \mathrm{CH}=\mathrm{CH}), 8.26\left(\mathrm{~s}, 1 \mathrm{H}, \mathrm{NH}\right.$, exchangeable with $\left.\mathrm{D}_{2} \mathrm{O}\right)$. Mass: $\mathrm{m} / \mathrm{z} 402\left([\mathrm{M}+1]^{+}, 4 \%\right)$. Calcd. for $\mathrm{C}_{21} \mathrm{H}_{24} \mathrm{FN}_{3} \mathrm{O}_{2} \mathrm{~S}$ (401.50). Calcd. C 62.82, H 6.03, N 10.47, S 7.99. Found: C 62.96; H, 6.11; N, 10.31; S, 7.85.

10) (E)- $N$-(3-methyl-1-(4-methylpiperazin-1-yl)-1-oxobutan-2-yl)-3-(thiophen-2yl)-acrylamide (5j)

TLC: $\mathrm{R}_{\mathrm{f}}=0.37$ [S $\mathrm{S}_{1}$, yield: $39 \%$ (Procedure B), m.p. $153^{\circ} \mathrm{C}-155^{\circ} \mathrm{C},[\alpha]^{25} \mathrm{D}(\mathrm{C}=0.5$, methanol $)=-12$. IR $v$ $\left(\mathrm{cm}^{-1}\right): 3376(\mathrm{NH}), 1669\left(\mathrm{C}=\mathrm{O}\right.$ amide). ${ }^{1} \mathrm{H} \quad \mathrm{NMR}$ (DMSO-d $\left.\mathrm{d}_{6}, \delta, \mathrm{ppm}\right): 0.98\left(\mathrm{~s}, 6 \mathrm{H}, 2 \mathrm{CH}_{3}\right), 2.21(\mathrm{~s}, 3 \mathrm{H}$, $\left.\mathrm{NCH}_{3}\right), 2.25-2.47(\mathrm{~m}, 1 \mathrm{H}, \mathrm{NHCHCH}), 2.54-2.66(\mathrm{~m}$, $4 \mathrm{H}, 2 \mathrm{CH}_{2}$, Pip-H), $3.21-3.32\left(\mathrm{~m}, 4 \mathrm{H}, 2 \mathrm{CH}_{2}\right.$, Pip-H), $4.47-4.58(\mathrm{~m}, 1 \mathrm{H}, \mathrm{NHCH}), 6.42(\mathrm{~d}, \mathrm{~J}=16 \mathrm{~Hz}, 1 \mathrm{H}$, $=\mathrm{CHCO}), 7.1(\mathrm{t}, \mathrm{J}=3 \mathrm{~Hz}, 1 \mathrm{H}, \mathrm{Th}-\mathrm{H} 4), 7.35(\mathrm{~d}, \mathrm{~J}=5 \mathrm{~Hz}$, $1 \mathrm{H}, \mathrm{Th}-\mathrm{H} 3), 7.48(\mathrm{~d}, 1 \mathrm{H}, \mathrm{J}=16 \mathrm{~Hz}, 1 \mathrm{H}, \mathrm{CH}=\mathrm{CH}), 7.63$ $(\mathrm{d}, \mathrm{J}=5.1 \mathrm{~Hz}, 1 \mathrm{H}, \mathrm{Th}-\mathrm{H} 5), 8.11(\mathrm{~s}, 1 \mathrm{H}, \mathrm{NH}$, exchangeable with $\mathrm{D}_{2} \mathrm{O}$ ). Mass: $\mathrm{m} / \mathrm{z} 335$ ([M] $]^{+}, 38 \%$ ). Calcd. for $\mathrm{C}_{17} \mathrm{H}_{25} \mathrm{~N}_{3} \mathrm{O}_{2} \mathrm{~S}$ (335.46). Calcd. C 60.87, H 7.51, N 12.53, S 9.56. Found: C, 60.99, H 7.59, N 12.41, S 9.46.

11) (E)- $N$-(1-(4-ethoxycarbonylpiperazin-1-yl)-3-methyl1-oxobutan-2-yl)-3-(thiophen-2-yl) acrylamide (5k)

TLC: $\mathrm{R}_{\mathrm{f}}=0.48$ [S $\mathrm{S}_{2}$, yield: $47 \%$ (Procedure C), m.p. $109^{\circ} \mathrm{C}-111^{\circ} \mathrm{C},[\alpha]^{25}{ }_{\mathrm{D}}(\mathrm{C}=0.5$, methanol $)=+17$. IR $v$ $\left(\mathrm{cm}^{-1}\right)$ : $3319(\mathrm{NH}), 1714(\mathrm{C}=\mathrm{O}$ ester $), 1657(\mathrm{C}=\mathrm{O}$ amide $)$. ${ }^{1} \mathrm{H}$ NMR (DMSO-d $\left.{ }_{6}, \delta, \mathrm{ppm}\right): 1.04\left(\mathrm{~s}, 6 \mathrm{H}, 2 \mathrm{CH}_{3}\right), 1.12$ $\left(\mathrm{t}, \mathrm{J}=7.1 \mathrm{~Hz}, 3 \mathrm{H}, \mathrm{CH}_{2} \mathrm{CH}_{3}\right), 1.87-1.93(\mathrm{~m}, 1 \mathrm{H}$, $\mathrm{NHCHCH}), 2.39-2.51\left(\mathrm{~m}, 4 \mathrm{H}, 2 \mathrm{CH}_{2}\right.$, Pip-H), $3.32-$ $3.43\left(\mathrm{~m}, 4 \mathrm{H}, 2 \mathrm{CH}_{2}\right.$, Pip-H), 4.07 (q, J =7.1 Hz, 2H, $\left.\mathrm{CH}_{2} \mathrm{CH}_{3}\right), 4.38-4.43(\mathrm{~m}, 1 \mathrm{H}, \mathrm{NHCH}), 6.46(\mathrm{~d}, \mathrm{~J}=16$ $\mathrm{Hz}, 1 \mathrm{H},=\mathrm{CHCO}), 7.1(\mathrm{t}, \mathrm{J}=3.2 \mathrm{~Hz}, 1 \mathrm{H}, \mathrm{Th}-\mathrm{H} 4), 7.36$ $(\mathrm{d}, \mathrm{J}=5 \mathrm{~Hz}, 1 \mathrm{H}, \mathrm{Th}-\mathrm{H} 3), 7.54(\mathrm{~d}, \mathrm{~J}=16 \mathrm{~Hz}, 1 \mathrm{H}$, $\mathrm{CH}=\mathrm{CH}), 7.87(\mathrm{~d}, \mathrm{~J}=5 \mathrm{~Hz}, 1 \mathrm{H}$, Th-H5), 8.09 (s, 1H, $\mathrm{NH}$, exchangeable with $\left.\mathrm{D}_{2} \mathrm{O}\right)$. Mass: $\mathrm{m} / \mathrm{z} 394\left([\mathrm{M}+1]^{+}\right.$, $3 \%$ ). Calcd. for $\mathrm{C}_{19} \mathrm{H}_{27} \mathrm{~N}_{3} \mathrm{O}_{4} \mathrm{~S}$ (393.50). Calcd. C 57.99, H 6.92, N 10.68, S 8.15. Found: C 57.83, H 6.85, N 10.75, S, 7.97.

12) (E)- $N$-(1-(4-(4-fluorophenyl)piperazin-1-yl)-3-methyl1-oxobutan-2-yl)-3-(thiophen-2-yl)acrylamide (51)

TLC: $\mathrm{R}_{\mathrm{f}}=0.42$ [S $\mathrm{S}_{1}$, yield: $53 \%$ (Procedure B), m.p. $139^{\circ} \mathrm{C}-141^{\circ} \mathrm{C},[\alpha]^{25}{ }_{\mathrm{D}}(\mathrm{C}=0.5$, methanol $)=+5$. IR $v$ $\left(\mathrm{cm}^{-1}\right)$ : $3450(\mathrm{NH}), 1672\left(\mathrm{C}=\mathrm{O}\right.$ amide). ${ }^{1} \mathrm{H}$ NMR (DMSO$\left.\mathrm{d}_{6}, \delta, \mathrm{ppm}\right): 0.98\left(\mathrm{~s}, 6 \mathrm{H}, 2 \mathrm{CH}_{3}\right), 1.97-2.12(\mathrm{~m}, 1 \mathrm{H}$, $\mathrm{NHCHCH}$ ), 2.26-2.37 (m, 4H, 2CH 2 , Pip-H), 3.38 - 3.59 (m, 4H, 2 $\mathrm{CH}_{2}$, Pip-H), 4.45 - 4.56 (m, 1H, NHCH), 6.6 $(\mathrm{d}, \mathrm{J}=15.6 \mathrm{~Hz}, 1 \mathrm{H},=\mathrm{CHCO}), 6.67(\mathrm{~m}, 2 \mathrm{H}, \mathrm{Ph}-\mathrm{H}), 6.98$ (m, 2H, Ph-H), 7.08 (t, J = 3 Hz, 1H, Th-H4), $7.23(\mathrm{~d}, \mathrm{~J}=$ $4.8 \mathrm{~Hz}, 1 \mathrm{H}, \mathrm{Th}-\mathrm{H} 3), 7.54$ (d, J = $15 \mathrm{~Hz}, 1 \mathrm{H}, \mathrm{CH}=\mathrm{CH})$, $7.65(\mathrm{~d}, \mathrm{~J}=4.8 \mathrm{~Hz}, 1 \mathrm{H}, \mathrm{Th}-\mathrm{H} 5), 8.09$ (s, 1H, NH, exchangeable with $\left.\mathrm{D}_{2} \mathrm{O}\right)$. Mass: $\mathrm{m} / \mathrm{z} 416\left([\mathrm{M}+1]^{+}, 5 \%\right)$. Calcd. for $\mathrm{C}_{22} \mathrm{H}_{26} \mathrm{FN}_{3} \mathrm{O}_{2} \mathrm{~S}$ (415.52). Calcd. C 63.59, H 6.31, N 10.11, S 7.72. Found: C 63.45, H 6.25, N 10.23, S 7.79.

13) (E)- $N$-(1-(4-methylpiperazin-1-yl)-1-oxo-3-phenylpropan-2-yl)-3-(thiophen-2-yl)acrylamide (5m)

TLC: $\mathrm{R}_{\mathrm{f}}=0.37$ [ $\mathrm{S}_{1}$ ], yield: $52 \%$ (Procedure B); m.p. $127^{\circ} \mathrm{C}-129^{\circ} \mathrm{C},[\alpha]^{25}(\mathrm{C}=0.5$, methanol $)=+25$. IR $v$ $\left(\mathrm{cm}^{-1}\right): 3376(\mathrm{NH}), 1669\left(\mathrm{C}=\mathrm{O}\right.$ amide). ${ }^{1} \mathrm{H}$ NMR (DMSO-d $\left.{ }_{6}, \delta, \mathrm{ppm}\right): 2.07$ (s, 3H, $\left.\mathrm{NCH}_{3}\right), 2.67-2.79(\mathrm{~m}$, $4 \mathrm{H}, 2 \mathrm{CH}_{2}$, Pip-H), 3.15 (brd, J = 5.5 Hz, $2 \mathrm{H}, \mathrm{CH}_{2} \mathrm{Ph}$ ), $3.28-3.37$ (m, 4H, 2CH $\mathrm{CH}_{2}$ PHz ip-H), 5.04 (t, J = $5 \mathrm{~Hz}$, $1 \mathrm{H}, \mathrm{NHCHCO}) 6.42(\mathrm{~d}, \mathrm{~J}=16,1 \mathrm{H},=\mathrm{CHCO}), 7.07-7.1$ (m, 5H, Ph-H), 7.14 (t, J = $3 \mathrm{~Hz}, 1 \mathrm{H}, \mathrm{Th}-\mathrm{H} 4), 7.25$ (d, J = $5.1 \mathrm{~Hz}, 1 \mathrm{H}, \mathrm{Th}-\mathrm{H} 3), 7.48(\mathrm{~d}, \mathrm{~J}=16 \mathrm{~Hz}, 1 \mathrm{H}, \mathrm{CH}=\mathrm{CH})$, $7.63(\mathrm{t}, \mathrm{J}=5.2 \mathrm{~Hz}, 1 \mathrm{H}$, Th-H5), $8.56(\mathrm{~s}, 1 \mathrm{H}, \mathrm{NH}, \mathrm{ex}-$ changeable with $\left.\mathrm{D}_{2} \mathrm{O}\right)$. Mass: $\mathrm{m} / \mathrm{z} 384\left([\mathrm{M}+1]^{+}, 15 \%\right)$. Calcd. for $\mathrm{C}_{21} \mathrm{H}_{25} \mathrm{~N}_{3} \mathrm{O}_{2} \mathrm{~S}$ (383.51). Calcd. C 65.77, H 6.57, N 10.96, S 8.36. Found: C 65.89, H 6.65, N 10.78, S 8.26 .

14) (E)- $N$-(1-(4-ethoxycarbonylpiperazin-1-yl)-1-oxo-3phenylpropan-2-yl)-3-(thiophen-2-yl)acrylamide (5n)

TLC: $\mathrm{R}_{\mathrm{f}}=0.48$ [S $\mathrm{S}_{2}$, yield: $47 \%$ (Procedure B), m.p. $113^{\circ} \mathrm{C}-115^{\circ} \mathrm{C},[\alpha]_{\mathrm{D}}^{25}(\mathrm{C}=0.5$, methanol $)=+15$. IR $v$ $\left(\mathrm{cm}^{-1}\right)$ : $3319(\mathrm{NH}), 1714(\mathrm{C}=\mathrm{O}$ ester $), 1657(\mathrm{C}=\mathrm{O}$ amide $)$. ${ }^{1} \mathrm{H}$ NMR (DMSO-d $\left.6, \delta, \mathrm{ppm}\right): 1.18(\mathrm{t}, \mathrm{J}=7.15 \mathrm{~Hz}, 3 \mathrm{H}$, $\mathrm{CH}_{2} \mathrm{CH}_{3}$ ), 3.17 (brd, J = 5 Hz, 2H, $\mathrm{CH}_{2} \mathrm{Ph}$ ), 2.59 - 2.71

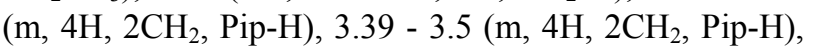
4.03 (q, J =7.1 Hz, 2H, $\left.\mathrm{CH}_{2} \mathrm{CH}_{3}\right), 5.04(\mathrm{t}, \mathrm{J}=5 \mathrm{~Hz}, 1 \mathrm{H}$, NHCHCO), $6.46(\mathrm{~d}, \mathrm{~J}=15 \mathrm{~Hz}, 1 \mathrm{H},=\mathrm{CHCO}), 7.07-7.09$ (m, 5H, Ph-H), 7.11 (t, J = 3 Hz, 1H, Th-H4), 7.36 (d, J = $5.1 \mathrm{~Hz}, 1 \mathrm{H}$, Th-H3), $7.57(\mathrm{~d}, \mathrm{~J}=15 \mathrm{~Hz}, 1 \mathrm{H}, \mathrm{CH}=\mathrm{CH})$, $7.87(\mathrm{~d}, \mathrm{~J}=5 \mathrm{~Hz}, 1 \mathrm{H}, \mathrm{Th}-\mathrm{H} 5), 8.59(\mathrm{~s}, 1 \mathrm{H}, \mathrm{NH}, \mathrm{ex}-$ changeable with $\left.\mathrm{D}_{2} \mathrm{O}\right)$. Mass: $\mathrm{m} / \mathrm{z} 442\left([\mathrm{M}+1]^{+}, 13 \%\right)$. Calcd. for $\mathrm{C}_{23} \mathrm{H}_{27} \mathrm{~N}_{3} \mathrm{O}_{4} \mathrm{~S}$ (441.54). Calcd. C 62.56, H 6.16, N 9.52, S 7.26. Found: C 62.69, H 6.24, N 9.43, S 7.18.

15) (E)-N-(1-(4-(4-fluorophenyl)piperazin-1-yl)-1-oxo-3phenylpropan-2-yl)-3-(thiophen-2-yl)acrylamide (5o)

TLC: $\mathrm{R}_{\mathrm{f}}=0.42$ [S $\mathrm{S}_{1}$, yield: $53 \%$ (Procedure B); m.p. $139^{\circ} \mathrm{C}-141^{\circ} \mathrm{C},[\alpha]^{25} \mathrm{D}(\mathrm{C}=0.5$, methanol $)=-27$. IR $v$ $\left(\mathrm{cm}^{-1}\right): 3370(\mathrm{NH}), \quad 1668 \quad(\mathrm{C}=\mathrm{O}$ amide $) .{ }^{1} \mathrm{H} \quad \mathrm{NMR}$ (DMSO-d $\left.{ }_{6}, \delta, \mathrm{ppm}\right): 2.79-2.90$ (m, $4 \mathrm{H}, 2 \mathrm{CH}_{2}$, Pip-H), 3.15 (brd, $\left.\mathrm{J}=5 \mathrm{~Hz}, 2 \mathrm{H}, \mathrm{CH}_{2} \mathrm{Ph}\right), 3.25-3.37(\mathrm{~m}, 4 \mathrm{H}$, $2 \mathrm{CH}_{2}$, Pip-H), 5.09 (t, J = 5 Hz, 1H, NHCHCO), 6.46 (d, $\mathrm{J}=15 \mathrm{~Hz}, 1 \mathrm{H},=\mathrm{CHCO}), 6.68(\mathrm{~m}, 2 \mathrm{H}, \mathrm{Ph}-\mathrm{H}), 6.97(\mathrm{~m}$, 2H, Ph-H), $7.0(\mathrm{~m}, 5 \mathrm{H}, \mathrm{Ph}-\mathrm{H}), 7.08(\mathrm{t}, \mathrm{J}=3.1 \mathrm{~Hz}, 1 \mathrm{H}$, Th-H4), $7.12(\mathrm{~d}, \mathrm{~J}=5 \mathrm{~Hz}, 1 \mathrm{H}, \mathrm{Th}-\mathrm{H} 3), 7.53$ (d, J = 15 $\mathrm{Hz}, 1 \mathrm{H}, \mathrm{CH}=\mathrm{CH}), 7.55$ (d, J = 5 Hz, 1H, Th-H5), 8.59 (s, $1 \mathrm{H}, \mathrm{NH}$, exchangeable with $\mathrm{D}_{2} \mathrm{O}$ ). Mass: $\mathrm{m} / \mathrm{z} 464$ $\left((\mathrm{M}+1]^{+}, 4 \%\right)$. Calcd. for $\mathrm{C}_{26} \mathrm{H}_{26} \mathrm{FN}_{3} \mathrm{O}_{2} \mathrm{~S}$ (463.57). Calcd. 
C 67.36, H 5.65, N 9.06, S 6.92. Found: C 67.42, H 5.69, $\mathrm{N} 9.00, \mathrm{~S} 6.88$.

16) (E)- $N$-(3-(4-hydroxyphenyl)-1-(4-methylpiperazin-1yl)-1-oxo-propan-2-Yl)-3-(thiophen-2-yl)acrylamide (5p)

TLC: $\mathrm{R}_{\mathrm{f}}=0.37\left[\mathrm{~S}_{1}\right.$ ], yield: $39 \%$ (Procedure B), $54 \%$ (Procedure C); m.p. $127^{\circ} \mathrm{C}-129^{\circ} \mathrm{C},[\alpha]^{25}(\mathrm{C}=0.5$, methanol $)=-100$. IR $v\left(\mathrm{~cm}^{-1}\right): 3376(\mathrm{NH}), 3215(\mathrm{br} \mathrm{OH})$, $1671\left(\mathrm{C}=\mathrm{O}\right.$ amide). ${ }^{1} \mathrm{H}$ NMR (DMSO-d $\left.\mathrm{d}_{6}, \delta, \mathrm{ppm}\right): 2.2$ (s, $3 \mathrm{H}, \mathrm{NCH}_{3}$ ), 2.56-2.67 (m, 4H, 2CH 2 , Pip-H), 2.82 (brd, J $\left.=5.5 \mathrm{~Hz}, 2 \mathrm{H}, \mathrm{PhCH}_{2}\right), 3.31-3.51\left(\mathrm{~m}, 4 \mathrm{H}, 2 \mathrm{CH}_{2}, \mathrm{Pip}-\mathrm{H}\right)$, $4.7(\mathrm{t}, \mathrm{J}=5.5 \mathrm{~Hz}, 1 \mathrm{H}, \mathrm{NHCHCO}), 6.47(\mathrm{~d}, \mathrm{~J}=16 \mathrm{~Hz}, 1 \mathrm{H}$, $=\mathrm{CHCO}), 6.84(\mathrm{~d}, \mathrm{~J}=6.7 \mathrm{~Hz}, 2 \mathrm{H}, \mathrm{Ph}-\mathrm{H}), 6.94(\mathrm{~d}, \mathrm{~J}=6.7$ $\mathrm{Hz}, 2 \mathrm{H}, \mathrm{Ph}-\mathrm{H}), 7.1(\mathrm{t}, \mathrm{J}=3.2 \mathrm{~Hz}, 1 \mathrm{H}, \mathrm{Th}-\mathrm{H} 4), 7.35(\mathrm{~d}, \mathrm{~J}$ $=5.2 \mathrm{~Hz}, 1 \mathrm{H}, \mathrm{Th}-\mathrm{H} 3), 7.57(\mathrm{~d}, \mathrm{~J}=16 \mathrm{~Hz}, 1 \mathrm{H}, \mathrm{CH}=\mathrm{CH})$, $7.63(\mathrm{~d}, \mathrm{~J}=5.2 \mathrm{~Hz}, 1 \mathrm{H}$, Th-H5), $8.38(\mathrm{~s}, 1 \mathrm{H}, \mathrm{NH}, \mathrm{ex}-$ changeable with $\left.\mathrm{D}_{2} \mathrm{O}\right), 9.15(\mathrm{~s}, 1 \mathrm{H}, \mathrm{OH}$, exchangeable with $\left.\mathrm{D}_{2} \mathrm{O}\right)$. Mass: $\mathrm{m} / \mathrm{z} 400\left([\mathrm{M}+1]^{+}, 10 \%\right)$. Calcd. for $\mathrm{C}_{21} \mathrm{H}_{25} \mathrm{~N}_{3} \mathrm{O}_{3} \mathrm{~S}$ (399.51). Calcd. C 63.13, H 6.31, N 10.52, S 8.03. Found: C 63.25, H 6.35, N 10.40, S 7.95.

17) (E)- $N$-(1-(4-ethoxycarbonylpiperazin-1-yl)-3-(4hydroxyphenyl)-1-oxopropan-2-yl)-3-(thiophen-2-yl)acrylamide $(5 \mathrm{q})$

TLC: $\mathrm{R}_{\mathrm{f}}=0.48\left[\mathrm{~S}_{2}\right.$ ], yield: $47 \%$ (Procedure B), m.p. $113^{\circ} \mathrm{C}-115^{\circ} \mathrm{C},[\alpha]_{\mathrm{D}}^{25}(\mathrm{C}=0.5$, methanol $)=+18$. IR $v$ $\left(\mathrm{cm}^{-1}\right)$ : $3450(\mathrm{NH}), 3215$ (br OH), $1734(\mathrm{C}=\mathrm{O}$ ester), $1678\left(\mathrm{C}=\mathrm{O}\right.$ amide). ${ }^{1} \mathrm{H}$ NMR (DMSO- $\left.\mathrm{d}_{6}, \delta, \mathrm{ppm}\right): 1.14(\mathrm{t}$, $\left.\mathrm{J}=7.2 \mathrm{~Hz}, 3 \mathrm{H}, \mathrm{CH}_{2} \mathrm{CH}_{3}\right), 2.76-2.87\left(\mathrm{~m}, 4 \mathrm{H}, 2 \mathrm{CH}_{2}\right.$, Pip-H), 3.15 - 3.27 (m, 6H, Ph- $\mathrm{CH}_{2}+2 \mathrm{CH}_{2}$, Pip-H), 4.06 $\left(\mathrm{q}, \mathrm{J}=7.2 \mathrm{~Hz}, 2 \mathrm{H}, \mathrm{CH}_{2} \mathrm{CH}_{3}\right), 4.94(\mathrm{t}, \mathrm{J}=5.5 \mathrm{~Hz}, 1 \mathrm{H}$, NHCHCO), $6.46(\mathrm{~d}, \mathrm{~J}=15 \mathrm{~Hz}, 1 \mathrm{H},=\mathrm{CHCO}), 6.65(\mathrm{~d}, \mathrm{~J}$ $=6.7 \mathrm{~Hz}, 2 \mathrm{H}, \mathrm{Ph}-\mathrm{H}), 7.03(\mathrm{~d}, \mathrm{~J}=6.7 \mathrm{~Hz}, 2 \mathrm{H}, \mathrm{Ph}-\mathrm{H}), 7.1$ $(\mathrm{t}, \mathrm{J}=3.1 \mathrm{~Hz}, 1 \mathrm{H}, \mathrm{Th}-\mathrm{H} 4), 7.36(\mathrm{~d}, \mathrm{~J}=5.1 \mathrm{~Hz}, 1 \mathrm{H}$, Th-H3), 7.57 (d, J = 15.5 Hz, 1H, CH=CH), 7.63 (d, J = $5.1 \mathrm{~Hz}, 1 \mathrm{H}, \mathrm{Th}-\mathrm{H} 5), 8.53$ (s, 1H, NH, exchangeable with $\left.\mathrm{D}_{2} \mathrm{O}\right), 9.25\left(\mathrm{~s}, 1 \mathrm{H}, \mathrm{OH}\right.$, exchangeable with $\left.\mathrm{D}_{2} \mathrm{O}\right)$. Mass: $\mathrm{m} / \mathrm{z} 458\left([\mathrm{M}+1]^{+}, 1 \%\right)$. Calcd. for $\mathrm{C}_{23} \mathrm{H}_{27} \mathrm{~N}_{3} \mathrm{O}_{5} \mathrm{~S}(457.54)$. Calcd: C 60.38, H 5.95, N 9.18, S 7.01. Found: C 60.23, H 5.87, N 9.34, S 6.93.

18) (E)- $N$-(1-(4-(4-fluorophenyl)piperazin-1-yl)-3-(4hydroxyphenyl)-1-oxopropan-2-yl)-3-(thiophen-2-yl)acrylamide (5r)

TLC: $\mathrm{R}_{\mathrm{f}}=0.47$ [S], yield $56 \%$ (Procedure B), m.p. $153^{\circ} \mathrm{C}-155^{\circ} \mathrm{C},[\alpha]_{\mathrm{D}}^{25}(\mathrm{C}=0.5$, methanol $)=-15$. IR $v$ $\left(\mathrm{cm}^{-1}\right)$ : $3222(\mathrm{br}, \mathrm{OH}), 3317(\mathrm{NH}), 1672(\mathrm{C}=\mathrm{O}) .{ }^{1} \mathrm{H}$ NMR (DMSO-d $\left.\mathrm{d}_{6}, \delta, \mathrm{ppm}\right): 2.74-2.85\left(\mathrm{~m}, 4 \mathrm{H}, 2 \mathrm{CH}_{2}\right.$, Pip-H), 2.91 - 2.98 (m, 2H, Ph- $\left.\mathrm{CH}_{2}\right), 3.45$ - 3.56 (m, 4H, $2 \mathrm{CH}_{2}$, Pip-H), $4.98-5.02(\mathrm{~m}, 1 \mathrm{H}, \mathrm{CH}), 6.48$ (d, J = 15 $\mathrm{Hz}, 1 \mathrm{H},=\mathrm{CHCO}), 6.78(\mathrm{~m}, 2 \mathrm{H}, \mathrm{Ph}-\mathrm{H}), 6.90(\mathrm{~m}, 2 \mathrm{H}$, $\mathrm{Ph}-\mathrm{H}), 6.97$ (m, 2H, Ph-H), $7.03-7.1(\mathrm{~m}, 3 \mathrm{H}, \mathrm{Ph}-\mathrm{H}+$ Th-H4), 7.23 (d, J = 2.5 Hz, 1H, Th-H3), 7.52 (d, J = 15 $\mathrm{Hz}, 1 \mathrm{H}, \mathrm{CH}=\mathrm{CH}), 7.57(\mathrm{~d}, \mathrm{~J}=5.1 \mathrm{~Hz}, 1 \mathrm{H}, \mathrm{Th}-\mathrm{H} 5), 8.55$ (s, $1 \mathrm{H}, \mathrm{NH}$, exchangeable with $\left.\mathrm{D}_{2} \mathrm{O}\right), 9.31(\mathrm{~s}, 1 \mathrm{H}, \mathrm{OH}$, exchangeable with $\left.\mathrm{D}_{2} \mathrm{O}\right)$. MS: $\mathrm{m} / \mathrm{z} 480\left([\mathrm{M}+1]^{+}, 45 \%\right)$. Calcd for $\mathrm{C}_{26} \mathrm{H}_{26} \mathrm{FN}_{3} \mathrm{O}_{3} \mathrm{~S}$ (479.17). Calcd: $\mathrm{C}$ 65.12, $\mathrm{H}$
5.46, N 8.76, S 6.69 \%. Found: C 65.26, H 5.52, N 8.65, $\mathrm{S} 6.61 \%$.

19) (E)- $N$-[(2-(4-methylpiperazin-1-yl)-2-oxoethylcarbamoyl)-methyl]-3-(thiophen-2-yl)acrylamide (5s)

TLC: $\mathrm{R}_{\mathrm{f}}=0.56[\mathrm{~S}]$, yield $58 \%$ (Procedure C), m.p. $170^{\circ} \mathrm{C}-172^{\circ} \mathrm{C}$. IR $v\left(\mathrm{~cm}^{-1}\right): 3330,3151(\mathrm{NH}), 1665$, $1651(\mathrm{C}=\mathrm{O}) .{ }^{1} \mathrm{H}$ NMR (DMSO-d $\left.\mathrm{d}_{6}, \delta, \mathrm{ppm}\right): 2.2(\mathrm{~s}, 3 \mathrm{H}$, $\left.\mathrm{CH}_{3}\right), 3.61-3.65$ (m, 4H, Pip-H), $3.89-3.92(\mathrm{~m}, 4 \mathrm{H}$, Pip-H), 4.1 (s, 2H, $\mathrm{CH}_{2}$ ), 4.25 (s, 2H, $\left.\mathrm{CH}_{2}\right), 6.64(\mathrm{~d}, 1 \mathrm{H}$, $\mathrm{J}=15 \mathrm{~Hz},=\mathrm{CHCO}), 7.13(\mathrm{t}, 1 \mathrm{H}, \mathrm{J}=5 \mathrm{~Hz}, \mathrm{Th}-\mathrm{H} 4), 7.14$ $(\mathrm{d}, 1 \mathrm{H}, \mathrm{J}=2.7 \mathrm{~Hz}, \mathrm{Th}-\mathrm{H} 3), 7.61(\mathrm{~d}, 1 \mathrm{H}, \mathrm{J}=16 \mathrm{~Hz}$, $\mathrm{CH}=\mathrm{CH}), 7.65(\mathrm{~d}, 1 \mathrm{H}, \mathrm{J}=4.5 \mathrm{~Hz}, \mathrm{Th}-\mathrm{H} 5), 8.12(\mathrm{~s}, \mathrm{br}$, $2 \mathrm{H}, 2 \mathrm{NH}, \mathrm{D}_{2} \mathrm{O}$-exchangeable). MS: m/z $351\left([\mathrm{M}+1]^{+}\right.$, $3 \%$ ). Calcd for $\mathrm{C}_{16} \mathrm{H}_{22} \mathrm{~N}_{4} \mathrm{O}_{3} \mathrm{~S}$ (350.44). Calcd: C 54.84, $\mathrm{H}$ 6.33 , N 15.99, S $9.15 \%$. Found: C 54.92, H 6.39, N 15.86 , S $9.23 \%$.

20) (E)- $N$-[(2-(4-ethoxycarbonylpiperazin-1-yl)-2-oxoethylcar-bamoyl)-methyl]-3-(thiophen-2-yl)acrylamide (5t)

TLC: $\mathrm{R}_{\mathrm{f}}=0.62$ [S], yield 42\% (Procedure B), m.p. $158^{\circ} \mathrm{C}-160^{\circ} \mathrm{C}$. IR $v\left(\mathrm{~cm}^{-1}\right): 3350,3156(\mathrm{NH}), 1664$, $1658(\mathrm{C}=\mathrm{O}) .{ }^{1} \mathrm{H}$ NMR (DMSO-d 6 , $\left.\delta, \mathrm{ppm}\right): 1.2(\mathrm{t}, 3 \mathrm{H}, \mathrm{J}$ $\left.=7 \mathrm{~Hz}, \mathrm{CH}_{2} \mathrm{CH}_{3}\right), 3.6(\mathrm{~m}, 4 \mathrm{H}, \mathrm{Pip}-\mathrm{H}), 3.9(\mathrm{~m}, 4 \mathrm{H}$, Pip-H), 4.1 (q, $\left.2 \mathrm{H}, \mathrm{J}=7 \mathrm{~Hz}, \mathrm{CH}_{2} \mathrm{CH}_{3}\right), 4.19$ (s, $2 \mathrm{H}, \mathrm{CH}_{2}$ ), $4.25\left(\mathrm{~s}, 2 \mathrm{H}, \mathrm{CH}_{2}\right), 6.62(\mathrm{~d}, 1 \mathrm{H}, \mathrm{J}=16 \mathrm{~Hz},=\mathrm{CHCO}), 7.1$ (t, $1 \mathrm{H}, \mathrm{J}=5 \mathrm{~Hz}, \mathrm{Th}-\mathrm{H} 4), 7.13(\mathrm{~d}, 1 \mathrm{H}, \mathrm{J}=2.5 \mathrm{~Hz}, \mathrm{Th}-\mathrm{H} 3)$, $7.51(\mathrm{~d}, 1 \mathrm{H}, \mathrm{J}=16 \mathrm{~Hz}, \mathrm{CH}=\mathrm{CH}), 7.7(\mathrm{~d}, 1 \mathrm{H}, \mathrm{J}=5 \mathrm{~Hz}$, Th-H5), 8.21 (s, br, 2H, 2NH, $\mathrm{D}_{2} \mathrm{O}-$-exchangeable). MS: $\mathrm{m} / \mathrm{z} 408\left([\mathrm{M}+1]^{+}, 54 \%\right)$. Calcd for $\mathrm{C}_{18} \mathrm{H}_{24} \mathrm{~N}_{4} \mathrm{O}_{5} \mathrm{~S}(430.5)$. Calcd: C 52.93, H 5.92, N 13.72, S $7.85 \%$. Found: C 52.82 , H 5.88, N 13.84, S $7.79 \%$.

21) (E)- $N$-((2-(4-(4-fluorophenyl)piperazin-1-yl)-2oxoethylcarbamoyl)-methyl)-3-(thiophen-2-yl)acrylamide $(5 \mathrm{u})$

TLC: $\mathrm{R}_{\mathrm{f}}=0.42\left[\mathrm{~S}_{1}\right]$, yield $53 \%$ (Procedure C), m.p. $190^{\circ} \mathrm{C}-192^{\circ} \mathrm{C}$. IR $v\left(\mathrm{~cm}^{-1}\right): 3377,3161(\mathrm{NH}), 1662$ $(\mathrm{C}=\mathrm{O}) .{ }^{1} \mathrm{H}$ NMR $\left(\right.$ DMSO-d $\left._{6}, \delta, \mathrm{ppm}\right): 3.1-3.12(\mathrm{~m}, 4 \mathrm{H}$, Pip-H), $3.55-3.60$ (m, 4H, Pip-H), $4.1\left(\mathrm{~s}, 2 \mathrm{H}, \mathrm{CH}_{2}\right)$, $4.25\left(\mathrm{~s}, 2 \mathrm{H}, \mathrm{CH}_{2}\right), 6.61(\mathrm{~d}, 1 \mathrm{H}, \mathrm{J}=16 \mathrm{~Hz},=\mathrm{CHCO}), 6.9$ (t, $1 \mathrm{H}, \mathrm{J}=5 \mathrm{~Hz}, \mathrm{Th}-\mathrm{H} 4), 7.02(\mathrm{~d}, 1 \mathrm{H}, \mathrm{J}=2.5 \mathrm{~Hz}, \mathrm{Th}-\mathrm{H} 3)$, $7.1(\mathrm{~m}, 2 \mathrm{H}, \mathrm{Ph}-\mathrm{H}), 7.23(\mathrm{~m}, 2 \mathrm{H}, \mathrm{Ph}-\mathrm{H}), 7.52(\mathrm{~d}, 1 \mathrm{H}, \mathrm{J}=$ $16 \mathrm{~Hz}, \mathrm{CH}=\mathrm{CH}), 7.75(\mathrm{~d}, 1 \mathrm{H}, \mathrm{J}=4.5 \mathrm{~Hz}, \mathrm{Th}-\mathrm{H} 5), 8.32$ (s, 2H, 2 NH- $\mathrm{D}_{2} \mathrm{O}$-exchangeable). MS: $\mathrm{m} / \mathrm{z} 430$ ([M] $\left.]^{+}, 7 \%\right)$. Calcd for $\mathrm{C}_{21} \mathrm{H}_{23} \mathrm{FN}_{4} \mathrm{O}_{3} \mathrm{~S}$ (430.5). Calcd: C 58.59, H 5.39, N 13.01, S $7.45 \%$. Found: C 58.50, H 5.26, N 13.23, S $7.39 \%$.

\section{Biological Activities}

Animals were obtained from the animal house colony of the National Research Center, Cairo, Egypt. All animals were allowed free access to water and were kept on a constant standard diet. All procedures involving animals were carried out in accordance with the guide for the care and use of laboratory animals (National Academy of 
Science of Egypt) and were approved by the Animals Studies Committee at Washington University. Adult male albino rats, weighing $150-180 \mathrm{~g}$, fasted for $12 \mathrm{~h}$ and used for determining the anti-inflammatory activity and $\mathrm{LD}_{50}$.

\subsection{Anti-Allergic Activity}

\subsubsection{Evaluation}

The percent inhibition of spasmogen induced contraction is calculated. The percentage of inhibition in contraction (due to histamine release) is the anti-allergic potency, which is compared to that of Loratadine (standard reference drug).

\subsubsection{Procedure}

Albino guinea pigs of either sex weighing 300 - $450 \mathrm{~g}$ are sacrificed with an overdose of ether. The chest cavity is opened and the lungs are removed. They were cut into strips of $5 \mathrm{~cm}$ and placed into a physiological saline solution. Thereafter, the lung strips are mounted in an organ bath containing a nutritive solution. The bath was bubbled with carbogen and maintained at $37^{\circ} \mathrm{C}$ under a preload of $0.5 \mathrm{~g}-3 \mathrm{~g}$; the tissue was left to equilibrate for 30 - 60 minutes. Prior to testing carbachol is added to the bath to test the lung strips ability of contraction. Twenty minutes later, two values are obtained by adding the spasmogen.

- Histamine dihydrochloride $10^{-6} \mathrm{~g} / \mathrm{ml}$ for 5 minutes,

- Ca-ionophore $5 \times 10^{-6} \mathrm{~g} / \mathrm{ml}$ for 5 minutes, or

- Leukotriene $\mathrm{LTC}_{4}\left(10^{-9}-10^{-8}\right) \mathrm{g} / \mathrm{ml}$ for 10 minutes,

To the bath and recording the contractile force at its maximal level. Following 20 minutes, equilibration period resulted, the spasmogen is administrated again, 5 minutes thereafter, and the test compound is added in cumulative dose from $10^{-8}-10^{-4} \mathrm{~g} / \mathrm{ml}$ at 5 or 10 minutes intervals. The contractile response is determined isometriccally [18].

\subsection{Evaluation of Anti-Inflammatory Activity}

\subsubsection{Protection against Edema}

The inhibitory activity of the studied compounds on carrageenan-induced rat's paw edema was carried out according to the method of Winter et al. [19,20]. Groups of adult male albino rats (150 - $180 \mathrm{gm})$, each of 8 animals were orally dosed with the test compounds at a dose level of 2.5 and $5 \mathrm{mg} / \mathrm{kg}$ an hour before carrageenan challenge. Foot paw edema was induced by sub planter injection of $0.05 \mathrm{ml}$ of $1 \%$ suspension of carrageenin in saline into the planter tissue of one hind paw. An equal volume of saline was injected to the other hind paw and served as control. Four hours after drug administration the animal was decapitated, blood was collected and the paws were rapidly excised.
The average weight of edema was estimated for the treated as well as the control group and the percentage inhibition of weight of edema was also evaluated then percentage protection against edema was estimated (Table 1). Indomethacin ( 2.5 and $5 \mathrm{mg} / \mathrm{kg}$ ) was employed as standard reference against which the test compounds were compared.

\subsubsection{Estimation of Plasma Prostaglandin $\mathrm{E}_{2}\left(\mathrm{PGE}_{2}\right)$}

Heparinized blood samples were collected from rats $(\mathrm{n}=$ 8), plasma was separated by centrifugation at $12000 \mathrm{~g}$ for $2 \mathrm{~min}$ at $4^{\circ} \mathrm{C}$ and immediately stored frozen $20^{\circ} \mathrm{C}$ until use.

The designs correlate-EIA prostaglandin in $\mathrm{E}_{2}\left(\mathrm{PGE}_{2}\right)$ kit is a competitive immune assay for the quantitative determination of $\mathrm{PGE}_{2}$ in biological fluids. The kit uses a monoclonal antibody to $\mathrm{PGE}_{2}$ to bind, in a competitive manner, the $\mathrm{PGE}_{2}$ in the sample. After a simultaneous incubation at room temperature the excess reagents were washed away and the substrate was added. After a short incubation time the enzyme reaction was stopped and the yellow color generated was read on a micro plate reader (DYNATCH, MR 5000) at $405 \mathrm{~nm}$. The intensity of the bound yellow color is inversely proportional to the concentration of $\mathrm{PGE}_{2}$ in either standard or samples. The percentage inhibition of plasma $\mathrm{PGE}_{2}$ for each compound was estimated [21] (Table 3).

\subsection{Evaluation of Acute Toxicity Study}

The test compounds were administered orally at different dose levels in separate groups of animals. After $24 \mathrm{~h}$ of drug administration percent mortality in each group was observed. From the data obtained, the lethal dose $\left(\mathrm{LD}_{50}\right)$ was calculated by the method of Smith [22].

\section{Results and Discussion}

\subsection{Chemistry}

The starting materials, $E$-3-(thiophen-2-yl)acrylamido) ester derivatives (3a-g) were obtained by reaction of $(E)$ 3-(thiophen-2-yl)acrylic acid (1) or (E)-3-(thiophen-2-yl) acryloyl chloride (2) with amino acid ester hydrochlorides of glycine, $\beta$-alanine, 4-aminobutyric acid and DValine, L-phenylalanine, L-tyrosine and the dipeptide glycylglycine. The products (3a-g) were prepared by the acid chloride method (procedure A), the 1,3-dicyclohexylcarbodiimide (DCCI) method (procedure B) and/or the mixed anhydride method (procedure C), followed by their alkaline hydrolysis under mild conditions to afford their corresponding acid derivatives (4a-g). The latter compounds were used as intermediates for the preparation of the targeted $N$-[(4-substituted-1-piperazinyl)-oxo(alkyl and ethylcarbamoyl)]-3-phenylacrylamides (5a-u) by their reactions with the appropriate piperazines by 
procedure B and/or procedure C as depicted in Scheme1. The structures of the synthesized products were confirmed in light of their IR, ${ }^{1} \mathrm{H}$ NMR and EI-MS spectral data, as well as, their elemental analyses (See experimental section). The infra red spectra indicated the expected absorption of the essential functional groups e.g. amide and C-terminal ester or carboxylic groups. Thus, the secondary amide nature of the compounds was represented by amide band I absorption at $1647-1673 \mathrm{~cm}^{-1}$, indicating a resonating $\mathrm{N}-\mathrm{C}=\mathrm{O}$ stretching vibrations. The obtained products were assigned $E$-configuration on the basis of their proton nuclear magnetic resonance spectra ( ${ }^{1} \mathrm{H}$ NMR), showed coupling constants of the olefinic protons $(J \approx 16-17 \mathrm{~Hz})$. The electron impact mass spectral data of the products were consistent with the proposed structures and showed molecular ion peaks corresponding to their molecular formulae.

\subsection{Pharmacology}

All the obtained products (5a-u) have been investigated for their anti-allergic and anti-inflammatory activeties. All tested products manifested highly $\mathrm{LD}_{50}$ values, so they are considered to be of high safety margins (Table 3).

Compounds 5j, 51, 5p, 5r, 5q, 5m, 5o, 5s, 5u, 5d, 5a and $5 \mathrm{c}$ showed to be of high activities relative to that of the reference drug Loratadine (Clorinix ${ }^{\circledR}$ ) (Table 3). It could be in relation with the presence of Valine (5j $98 \%$ and 51 97\%) and tyrosine (5p 96\%, 5r 94\% and 5q 90\%) residues, also, the methyl piperazine segment showed to be more active than 4-fluorophenyl and 4-ethoxycarbonyl one (Table 3).

The evaluation of anti-inflammatory activity was carried out according to the method of winter et al, where

Table 3. Anti-allergy and anti-inflammatory activities of compounds (5g-u).

\begin{tabular}{|c|c|c|c|c|}
\hline Compd. No. & $\begin{array}{l}\% \text { inhibition } \\
\text { in contraction }\end{array}$ & $\begin{array}{c}\% \text { protection against edema at } \\
2 \mathrm{mg} / \mathrm{kg} \text { dose level }\end{array}$ & $\begin{array}{l}\% \text { inhibition of PEG2 at } \\
2 \mathrm{mg} / \mathrm{kg} \text { dose level }\end{array}$ & $\begin{array}{c}\mathrm{LD}_{50} \\
\mathrm{mg} / \mathrm{Kg}\end{array}$ \\
\hline $5 \mathrm{a}$ & $75 \pm 0.39$ & 70.77 & 60.15 & 613.44 \\
\hline $5 b$ & $46 \pm 0.09$ & 45.16 & 34.12 & 264.48 \\
\hline $5 \mathrm{c}$ & $71 \pm 0.35$ & 69.18 & 59.55 & 2578.34 \\
\hline $5 \mathrm{~d}$ & $78 \pm 0.39$ & 40.55 & 29.15 & 268.4 \\
\hline $5 \mathrm{e}$ & $56 \pm 0.07$ & $\mathrm{NA}^{*}$ & NA & 241.3 \\
\hline $5 \mathrm{f}$ & $67 \pm 0.22$ & 42.12 & 32.16 & 123.4 \\
\hline $5 \mathrm{~g}$ & NA & $98.16 \pm 0.23$ & $83.27 \pm 0.37$ & $246.6 \pm 2.33$ \\
\hline $5 \mathrm{~h}$ & NA & $81.9 \pm 0.13$ & $63.5 \pm 0.14$ & $462.4 \pm 2.1$ \\
\hline $5 \mathrm{i}$ & NA & $90.8 \pm 0.19$ & $76.6 \pm 0.15$ & $123.4 \pm 1.6$ \\
\hline $5 \mathrm{j}$ & $98 \pm 0.89$ & NA & NA & $2648 \pm 4.45$ \\
\hline $5 \mathrm{k}$ & $53 \pm 0.05$ & NA & NA & $2352 \pm 4.67$ \\
\hline 51 & $97 \pm 0.09$ & NA & NA & $2413 \pm 3.44$ \\
\hline $5 \mathrm{~m}$ & $89 \pm 0.44$ & NA & NA & $2684 \pm 5.33$ \\
\hline $5 n$ & $68 \pm 0.34$ & NA & NA & $254.66 \pm 2.56$ \\
\hline 50 & $88 \pm 0.55$ & NA & NA & $2678.34 \pm 4.44$ \\
\hline $5 p$ & $96 \pm 0.23$ & NA & NA & $2356 \pm 5.33$ \\
\hline $5 q$ & $90 \pm 0.12$ & NA & NA & $2684.3 \pm 2.44$ \\
\hline $5 \mathrm{r}$ & $94 \pm 0.09$ & NA & NA & $163 \pm 2.9$ \\
\hline $5 \mathrm{~s}$ & $84 \pm 0.34$ & NA & NA & $2167.74 \pm 3.56$ \\
\hline $5 \mathrm{t}$ & $68 \pm 0.44$ & NA & NA & $2578.34 \pm 6.48$ \\
\hline $5 \mathrm{u}$ & $80 \pm 0.34$ & NA & NA & $2376.55 \pm 3.56$ \\
\hline Loratadine & 71 & - & - & - \\
\hline Indomethacine & - & 100 & 80 & - \\
\hline
\end{tabular}

"NA = no activity. 


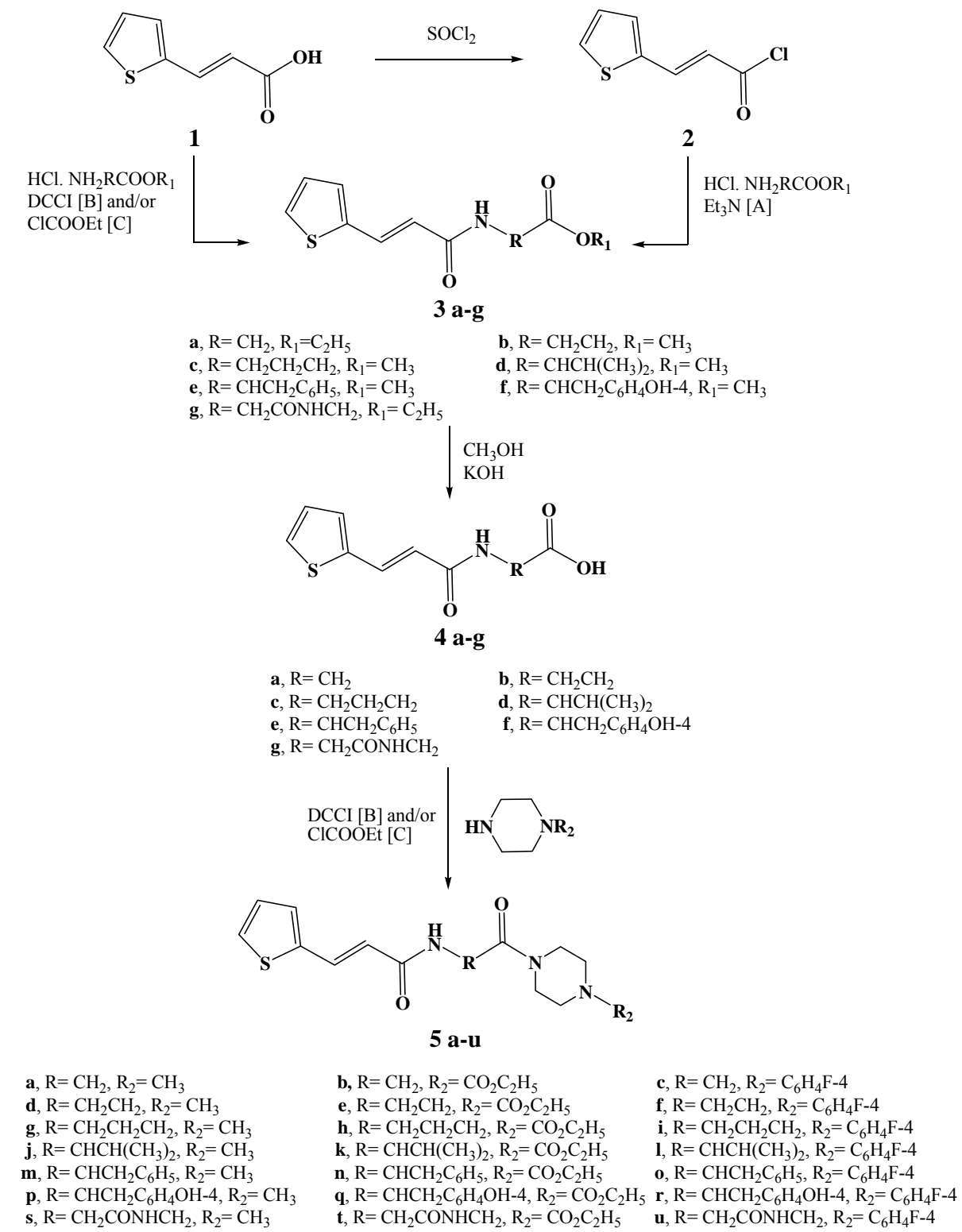

Scheme 1. Synthesis of N-[(4-substituted-1-piperazinyl)-oxo-(alkyl and ethylcarbamoyl)]-3-(2-thiophenyl)acrylamides (5a-u).

the inhibitory activity of the studied compounds on carrageenan-induced rat's paw edema. Most of these compounds have no anti-inflammatory activities except $5 \mathrm{~g}$ which showed the relative highest activities of $98 \%(\%$ protection against edema) and 83\% (\% inhibition of PEG2) in comparison with the reference drug Indomethacine.

\section{Conclusion}

A series of 21 products from the coupling of three piperazine derivatives with seven 3-(thiophen-2-yl) acrylamide amino acids was designed based on the concept of Nishikawa et al. [Figure 1, structure A]. Our novel design depends on the replacement of the aliphatic chain with the known biologically active amino acid segments. The obtained pharmacological screening results, showed that the presence of the amino acid residues in the products as linkers between 2-thiopheneacrylic acid and piperazine derivatives [Figure 1, structure B], enhance their anti-allergy activity and has no effect on increasing their anti-inflammatory one. All tested products manifested highly $\mathrm{LD}_{50}$ values, so they are considered to be of high safety margins.

\section{REFERENCES}

[1] F. J. Tinney, W. A. Cetenko, J. J. Kerbleski, D. T. Connor, R. J. Sorenson and D. J. Herzig, "Synthesis and Antiallergy Activity of 4-Oxo-4H-Pyrido[1,2-a]Thieno[2,3-d]- 
Pyrimidine," Journal of Medicinal Chemistry, Vol. 24, No. 7, 1981, pp. 878-882. doi:10.1021/jm00139a021

[2] C. A. Kontogiorgis and D. J. Hadjipavlou-Litina, "Non Steroidal Anti-Inflammatory and Anti-Allergy Agents," Current Medicinal Chemistry, Vol. 9, No. 1, 2002, pp. 8998. doi:10.2174/0929867023371409

[3] E. Nettis, M. C. Colanardi, A. Ferannini and A. Tursi, "Antihistamines as Important Toolls for Regulating Inflammation," Current Medicinal Chemistry-Anti-Inflammatory \& Anti-Allergy Agents, Vol. 4, No. 1, 2005, pp. 81-89. doi:10.2174/1568014053005372

[4] Y. Nishikawa, T. Shindo, K. Ishii, H. Nakamura, T. Kon and H. Uno, "cryamide Derivatives as Antiallergic Agents. I. Synthesis and Structure-Activity Relationships of $N$ [(4-Substituted 1-Piperazinyl)Alkyl]-3-(Aryl and Heteroaryl) Acryamides," Chemical \& Pharmaceutical Bulletin, Vol. 37, No. 1, 1989, pp. 100-105. doi:10.1248/cpb.37.100

[5] Y. Nishikawa, T. Shindo, K. Ishii, H. Nakamura, T. Kon and H. Uno, "Acrylamide Derivatives as Antiallergic Agents. 2. Synthesis and Structure-Activity Relationships of $N$ [4-[4-(Diphenylmethyl)Piperazinyl]-Butyl]-3-(3-Pyridyl) Acrylamides," Journal of Medicinal Chemistry, Vol. 32, No. 3, 1989, pp. 583-593. doi:10.1021/jm00123a012

[6] Y. Nishikawa, T. Shindo, K. Ishii, H. Nakamura, T. Kon and H. Uno, "Acrylamide Derivatives as Antiallergic Agents. III. Synthesis and Structure-Activity Relationships of $\mathrm{N}$ [4-(4-(Diphenylmethyl-1-Piperazinyl)-Butyl]- and $\mathrm{N}$-[4(4-(Diphenylmethylene-1-Piperidyl)-Butyl]-3-Heteroacrylamides," Chemical \& Pharmaceutical Bulletin, Vol. 37, No. 3, 1989, pp. 684-687. doi:10.1248/cpb.37.684

[7] K. Ishii, I. Yakuo, S. Motoyoshi, H. Nakagawa and H. Nakamura, "Inhibition of Leukotriene Production by $\mathrm{N}$-[4-[4-(Diphenylmethyl)-1-Piperazinyl]Butyl]-3-(6-Met hyl-3-Pyridyl)Acrylamide (AL-3264, a New Antiallergic Agent," The Japanese Journal of Pharmcology, Vol. 65, No. 1, 1994, pp. 19-25. doi:10.1254/jjp.65.19

[8] L. Chaitow and D. O. Chaitow, "Amino Acids in Therapy, A Guide to the Therapeutic Applications of Protein Constituents," Leon Chaitow-Thorsons Publishers Limited Inc., New York, 1985.

[9] C. Döbler, H. J. Kreuzfeld, H. W. Krause and M. Michalik, "Unusual Amino Acids IV. Assymmetric Synthesis of Thienylalanines," Tetrahedron: Assymetry, Vol. 4, No. 8, 1993, pp. 1833-1842. doi:10.1016/S0957-4166(00)80424-3

[10] S. J. Hodson, E. C. Bigham, D. S. Duch, G. K. Smith and R. Ferone, "Thienyl and Thiazolyl Acyclic Analogs of 5-Deazatetrahydrofolic Acid," Journal of Medicinal Chemistry, Vol. 37, No. 13, 1994, 2112-2115. doi:10.1021/jm00039a025

[11] J. Meiwes, M. Schudok and G. Kretzschmar, "Asymmetric Synthesis of L-Thienylalanines," Tetrahedron: Assymetry, Vol. 8, No. 4, 1997, pp. 527-536.

[12] T. Nagano, T. Hirano and M. Hirobe, "Superoxide Dis- mutase Mimics Based on Iron in Vivo," The Journal of Biological Chemistry, Vol. 264, No. 16, 1989, pp. 92439249.

[13] M. Baudry, S. Etienne, M. Bruce, M. Palucki, E. Jacobsen and B. Malfroy, "Salen-Manganese Complexes Are Superoxide Dismutase-Mimics," Biochemical and Biophysical Research Communications, Vol. 192, No. 2, 1993, pp. 964-968. doi:10.1006/bbrc.1993.1509

[14] S. Guner, S. Karaböcek and I. Kaklikkaya, "Models for Superoxide Dismutases: Characterization of Mononuclear $\mathrm{Cu}(\mathrm{II}), \mathrm{Fe}(\mathrm{III})$, and $\mathrm{Mn}(\mathrm{II})$ Complexes with 4',5'-Bis (Salicylideneimino)Benzo-15-Crown-5," Bioorganic \& Medicinal Chemistry, Vol. 7, No. 2, 1999, pp. 329-333. doi:10.1016/S0968-0896(98)00240-5

[15] A. M. Shalaby and W. I. El-Eraqi, "Studies on $O$ [2,6-Dichlorophenyl-1-Amino]Phenyl Acetic Acid I. Synthesis, Antiinflammatory, Analgesic and Ulcerogenic Activities of Some New Amino Acid Conjugates," Il Farmaco, Vol. 52, No. 2, 1997, 83-87.

[16] M. H. Abo-Ghalia, A. M. Shalaby, W. I. El-Eraqi and H. M. Awad, "Synthesis and Anti-Phlogistic Potency of Some New Non-Proteiogenic Amino Acid Conjugates of 'Diclofenac'," Amino Acids, Vol. 16, No. 3-4, 1999, pp. 425-440. doi:10.1007/BF01388181

[17] A. M. Shalaby, A. A. El-Sawy, O. I. Abd El-Salam, M. Abdullah and S. A. Elshihaby, "Amino Acid and Peptide Derivatives as Anti-Allergic and Anti-Inflammatory Agents," Egyptian Journal of Chemistry, Vol. 51, 2008, pp. 807821.

[18] J. C. Ferman, R. Shelly and S. E. Webber, "Contraction of Guinea-Pig Lung Parenchymal Strips by Substance P and Related Peptides," Archives Internationales de Pharmacodynamie et de Thérapie, Vol. 278, No. 2, 1985, pp. 193-206.

[19] C. A. Winter, E. A. Risely and G. W. Nuss, "CarrageeninInduced Edema in Hind Paw of the Rat as an Assay for Antiiflammatory Drugs," Proceedings of the Society for Experimental Biology and Medicine, Vol. 111, 1962, pp. 544-547.

[20] C. A. Winter, E. A. Risely and G. W. Nuss, "Anti-Inflammatory and Antipyretic Activities of Indomethacin, 1-(p-Chlorobenzoyl)-5-Methoxy-2-Methylindole-3-Aceti c acid," Journal of Pharmacology and Experimental Therapeutics, Vol. 141, No. 3, 1963, pp. 369-376.

[21] F. Herrmann, A. Lindemann, J. Gauss and R. Mertelsmann, "Cytokinestimulation of Prostaglandin Synthesis from Endogenous And Exogenous Arachidonic Acids in Polymorphonuclear Leukocytes Involving Activation and New Synthesis of Cyclooxygenase," European Journal of Immunology, Vol. 20, No. 11, 1990, pp. 2513-2516. doi:10.1002/eji.1830201124

[22] Q. E. Smith, "Pharmacological Screening Tests Progress in Medicinal Chemistry," Vol. 1, Butterworth, London, 1960 . 